\title{
Title: Bimodal neuromodulation combining sound and tongue stimulation reduces tinnitus symptoms in a large randomized clinical study
}

\author{
Authors: \\ Brendan Conlon ${ }^{1,2,3}$, Berthold Langguth ${ }^{4,5}$, Caroline Hamilton ${ }^{1}$, Stephen Hughes ${ }^{1}$, Emma Meade ${ }^{1}$, \\ Ciara O Connor ${ }^{1}$, Martin Schecklmann ${ }^{4,5}$, Deborah A Hall ${ }^{6,7,8}$, Sven Vanneste ${ }^{9,10}$, Sook Ling \\ Leong ${ }^{1,10}$, Thavakumar Subramanian ${ }^{3}$, Shona D'Arcy ${ }^{1}$, Hubert H Lim ${ }^{1,11,12, *}$
}

\footnotetext{
Affiliations:

${ }^{1}$ Neuromod Devices Limited, D08 R2YP, Ireland.

${ }^{2}$ School of Medicine, Trinity College Dublin, D02 R590, Ireland.

${ }^{3}$ Department of Otolaryngology, St. James's Hospital, D08 NHY1, Ireland.

${ }^{4}$ Department of Psychiatry and Psychotherapy, University of Regensburg, 93053, Germany.

${ }^{5}$ Interdisciplinary Tinnitus Center of the University of Regensburg, 93053, Germany.

${ }^{6}$ National Institute for Health Research Nottingham Biomedical Research Centre, NG7 2UH, UK.

${ }^{7}$ Hearing Sciences, Division of Clinical Neuroscience, University of Nottingham, NG7 2RD, UK.

${ }^{8}$ University of Nottingham Malaysia, Selangor Darul Ehsan, 43500, Malaysia.

${ }^{9} \mathrm{Lab}$ for Clinical and Integrative Neuroscience, School of Behavioral and Brain Sciences, The University of Texas at Dallas, 75080, USA.

${ }^{10}$ Global Brain Health Institute, Trinity College Dublin, D02 PN40, Ireland.

${ }^{11}$ Department of Otolaryngology - Head and Neck Surgery, University of Minnesota, 55455, USA.

${ }^{12}$ Department of Biomedical Engineering, University of Minnesota, 55455, USA.
}

*Corresponding author. Email: hubert.lim@neuromoddevices.com 


\title{
One Sentence Summary:
}

Noninvasive bimodal neuromodulation reduces tinnitus symptom severity within 12 weeks of treatment for up to 12 months after completing treatment

Overline: TINNITUS

\begin{abstract}
:
Tinnitus is a phantom auditory perception coded in the brain that can be bothersome or debilitating, affecting $10-15 \%$ of the population. Currently, there is no clinically recommended drug or device treatment for this major health condition. Animal research has revealed that sound paired with electrical somatosensory stimulation can drive extensive plasticity within the brain for tinnitus treatment. To investigate this bimodal neuromodulation approach in humans, we evaluated a noninvasive device that delivers sound to the ears and electrical stimulation to the tongue in a randomized, double-blinded, exploratory study that enrolled 326 adults with chronic subjective tinnitus. Participants were randomized into three parallel arms with different stimulation settings. Clinical outcomes were evaluated over a 12 -week treatment period and a 12 -month post-treatment phase. For the primary endpoints, participants achieved a statistically significant reduction in tinnitus symptom severity at the end of treatment based on two commonly used outcome measures, Tinnitus Handicap Inventory (Cohen's d effect size: -0.87 to -0.92 across arms; $P<0.001$ ) and Tinnitus Functional Index $(-0.77$ to $-0.87 ; P<0.001)$. Therapeutic improvements continued for 12 months post-treatment for specific bimodal stimulation settings, which had not previously been demonstrated in a large cohort for a tinnitus intervention. The treatment also achieved high compliance and satisfaction rates with no treatment-related serious adverse events. These positive therapeutic and long-term results motivate further clinical trials towards establishing bimodal neuromodulation as the first clinically recommended device treatment for tinnitus.
\end{abstract}




\section{Introduction}

The brain is a complex and robust network of cells that mediates our everyday functions from sensations and movements to consciousness and learning. In certain circumstances, the brain enters deviant states that can lead to debilitating or even life-threatening conditions (1-5). Neuromodulation has emerged as a promising approach to treat many different brain disorders, in which electrical or magnetic stimulation of the nervous system can be used to modulate aberrant neurons to improve a patient's symptoms (6-10). One widely used approach applies surface electrical stimulation on the scalp or body, including activation of the trigeminal or vagus nerve, to treat a wide range of health conditions, such as tinnitus, seizures, depression, anxiety, memory loss, inflammation and pain $(6-9,11-16)$. There is a growing consensus that this type of stimulation activates or modulates a broad area of the brain; however, greater or more consistent efficacy may be achieved through electrical stimulation paired with a targeted input to modulate specific brain regions for improving symptoms $(7,8,17-23)$. In particular, electrical stimulation applied to the scalp leads to current that spreads across the skull and activates a broad volume of the brain, or non-invasive electrical stimulation of a peripheral nerve can excite widespread projections throughout the brain that broadly activate multiple interconnected neural regions. A targeted input (e.g., a pure tone or movement of a finger) can be designed to activate a local population of neurons that overlaps with the broad activation caused by surface electrical stimulation; thus, this coordinated activation by both modalities can lead to enhanced modulation or paired plasticity within just the overlapping localized region $(7,8,17-23)$.

Paired stimulation has recently shown promise for treating a brain condition known as tinnitus, which is a phantom sound perception that occurs without any external acoustic input. Tinnitus can be bothersome or debilitating for $10-15 \%$ of the population, and is a major health issue in society (24-27). There is still no clinically recommended device or drug treatment for this condition $(28,29)$. Animal research and pilot human studies have shown that bimodal neuromodulation combining sound and electrical somatosensory stimulation (30), including tongue stimulation $(17,31)$, can drive extensive plasticity in the auditory system and improve tinnitus symptoms. Electrical somatosensory stimulation can broadly activate or modulate neurons throughout the auditory pathway, from the brainstem up to the auditory cortex, and in emotional and attentional centers $(13,17,30,32-40)$. Pairing specific acoustic and somatosensory stimuli can drive enhanced modulation within brain regions linked to tinnitus $(17,30,31)$, in which repeating stimulation of two inputs can lead to long-term therapeutic plasticity $(7,8,17,20,22,23)$.

There is a globally recognized clinical need for new treatments for tinnitus due to the lack of effective options for this debilitating condition. Based on the positive efficacy and safety outcomes in a previous human pilot study presenting sound and tongue stimulation to treat tinnitus (31), along with supporting animal data (17), we pursued a double-blind randomized clinical study (41) (clinicaltrials.gov, NCT02669069) to further evaluate different parameter settings of bimodal neuromodulation in a broad tinnitus population. Bimodal stimulation was delivered using a medical device (referred to as MBT in the study; marketed as Lenire) that pairs sound with electrical stimulation of the tongue (Fig. 1A). The main objective of this exploratory study was to build further supporting data for the safety and efficacy of bimodal neuromodulation. More 
specifically, the study aimed to evaluate the effects of three different parameter settings of bimodal stimulation on tinnitus symptoms, both in the general tinnitus population and in subtypes of patients that may be differentially responsive to the tested parameters. A large number of tinnitus participants were screened, randomized and enrolled into one of three study arms (Fig. 1B).

One challenge with these types of studies is the large range of parameters that can be evaluated for a treatment paradigm such as our bimodal neuromodulation, including types of acoustic stimuli, electrical pulse patterns and their location on the tongue, and durations and timings of stimulation. Based on previous animal and human research $(30,42)$, effective therapeutic benefit with bimodal neuromodulation may be achieved by implementing a specific delay between sound and electrical somatosensory stimulation, as well as matching the frequency spectrum of the acoustic stimulus to the tinnitus percept. Other studies in animals and tinnitus patients $(17,31,43)$ suggest that a broad spectrum of acoustic frequencies and/or a range of delays between sound and electrical somatosensory stimulation can be effective in driving extensive plasticity within auditory regions to alleviate tinnitus symptoms. Considering the parameters that could be effective for bimodal neuromodulation, attempting to investigate the contribution of each parameter with a sufficient number of subjects would require thousands of participants. Our strategy was to reduce the parameter space into three groups, in which several stimulation parameters differed between groups. The advantage of this approach is that multiple parameters can be explored with fewer treatment arms and a smaller sample size. The disadvantage of this approach is that if a significant difference is observed between treatment groups, then distinguishing the main determinant of the therapeutic effect would require a follow-up study. As this is the first in a series of planned clinical studies exploring the parameter space of bimodal neuromodulation for tinnitus treatment, we pursued this three arm study design to initially narrow in on a smaller and more manageable parameter space that would then be systematically evaluated in future clinical trials.

The study was conducted at two clinical sites: the Wellcome Trust-HRB Clinical Research Facility, St. James's Hospital, Dublin, Ireland and the Tinnituszentrum of the University Regensburg, Germany. Tinnitus participants were provided and instructed to use a bimodal neuromodulation device for 60 minutes daily for 12 weeks. They were clinically evaluated at 6 weeks and at 12 weeks during the treatment period (Fig. 1B). When treatment was completed, participants were assessed at three follow-up visits up to 12 months after returning their device. The primary endpoints of the study comprised of within-arm and between-arm comparisons of tinnitus symptom severity during treatment. The outcome measures utilized two widely used and validated instruments to assess changes in tinnitus symptom severity: Tinnitus Handicap Inventory (THI) and Tinnitus Functional Index (TFI) (44-47). Long-term changes in tinnitus symptom severity were also assessed during the 12-months post-treatment phase. Exploratory analyses described in the published protocol paper (41), including the effects of different stimulation parameters on subtypes of tinnitus participants and comparisons of alternative methods for measuring the impact of tinnitus, will be completed and presented in a subsequent publication. 


\section{Results}

\section{Characteristics and summary of study participants}

As previously described in the published protocol paper (41), study participants were recruited to the clinical trial using media advertising, and directed to an online eligibility assessment. Eligibility was initially assessed through a set of general pre-screening questions to manage the large number of candidates expected to respond to the advertising (48). As anticipated, a substantial number of candidates $(7,627)$ registered their interest in the trial. 5826 individuals completed the online assessment and 698 were invited for screening at a clinical site. Based on the inclusion criteria, 333 individuals were randomized into the three study arms (1:1:1), with 326 successfully completing enrollment and device fitting. Figure 2 presents the participant flow diagram for the study.

Enrolled participants were randomized into the three treatment arms according to four binary categories of stratification (41) using the method of minimization (49), as shown in Table 1. The four stratification categories included atonal tinnitus, hyperacusis, normal hearing and noise induced hearing loss. The participants were distributed across treatment arms with no significant differences $(P>0.05)$ in relation to the four stratification categories. There were also no significant differences $(P>0.05)$ across arms for the other characteristics and groups shown in Table 1. In addition to the stratification categories, the "Other Groups" listed in Table 1 were intentionally included in the study design to perform exploratory analyses and identify different subtypes of tinnitus patients who could be differentially responsive to certain bimodal stimulation parameters. These subtyping results are outside the scope of this paper and will be presented in a subsequent publication.

As listed in Table 1, 84.0\% of enrolled participants returned for their final visit at the end of their 12-week treatment. The high retention rate is mirrored in the high treatment compliance rate of $83.7 \%$ (fig. S1). Minimum treatment compliance was defined as device usage of at least 36 hours over the intended 12-week treatment period, which was specified in the design of the clinical trial (41) and derived from a previous bimodal neuromodulation study (31). The device logged the time and date of daily usage by each participant. There were no significant differences in the compliance rate or number of participants at the final visit between treatment arms $(P>0.05$; fig. S1). Participants also generally attended their visits at the intended time points specified in the study design (fig. S2).

Overall, there were no major differences in characteristics across study arms that would bias the primary endpoint analyses. There was also a high number of treatment-compliant participants that could be included into the primary endpoint analyses.

\section{Within-arm and between-arm therapeutic effects during treatment}

Each participant was fitted with a device delivering one of three bimodal stimulation settings (PS1, PS2, PS3; Fig. 1B). In a previous pilot study (31), paired sound and electrical tongue stimulation provided significant improvements in tinnitus symptoms. To explore which 
stimulation parameters contribute to the therapeutic effects, three different stimulation settings were designed for this study. All stimuli were presented at supra-threshold intensities, as in the previous pilot study, which enabled blinding of participants and researchers to the treatment arms. Details and schematics of the different stimulation settings are provided in the Materials and Methods and table S1.

PS1 consisted of a wide range of pure tones $(500-8000 \mathrm{~Hz})$ synchronized in time with a burst of electrical pulses delivered to the tongue surface. Since a wideband noise component was included in the paired stimuli used in the previous pilot study, the acoustic stimulus in PS1 also incorporated a broadband noise background. Previous animal research suggests there may be a specific delay or range of delays between sound and somatosensory stimulation for driving greater improvements in tinnitus $(17,30,42)$. However, it is not yet known how the effective delays identified in animals translate to humans. Presenting two stimuli synchronously is consistent with the concept of paired plasticity $(7,8,18-21)$, where a person can perceptually integrate and associate meaning between two inputs that are physically presented at the same time $(50,51)$. In keeping consistent with the paired plasticity approach, we presented acoustic and tongue stimuli at the same time for PS1. PS2 was similar to PS1, except there were short inter-stimulus delays between sound and tongue stimulation that were randomly distributed between 30 and $50 \mathrm{~ms}$. PS1 also had a fixed relationship between pure tone frequencies and stimulation locations on the tongue array, whereas PS2 did not have a fixed tone-to-tongue mapping. The rationale for PS2 was to assess if therapeutic effects required a specific acoustic feature to be systematically and synchronously mapped to a specific tongue location for a given session, as occurred in the previously published pilot study (31). The 30-50 ms range was selected so that the onset of the electrical pulses fell midway between the onset of the preceding and proceeding acoustic stimuli (i.e., for a repetition period of $\sim 80 \mathrm{~ms}$ ). Considering that an offset of $30-50 \mathrm{~ms}$ may not be sufficient to disrupt paired plasticity on a higher cortical or cognitive level that could function on longer time scales, greater inter-stimulus delays $(550-950 \mathrm{~ms})$ were introduced in PS3. These longer delays also required a longer repetition period, which was set to $\sim 2 \mathrm{~s}$ for PS 3 and resulted in a stimulation rate that was about 25 times less than the rate used for PS1 and PS2. To further assess if the frequency range of acoustic stimuli influenced therapeutic effects, only low frequency pure tones $(100-500 \mathrm{~Hz})$ were presented in PS3. Similar to PS2, PS3 did not have a fixed tone-to-tongue mapping.

During device fitting, sound volume was adjusted to each participant's hearing thresholds and electrical stimulation intensity was adjusted to each participant's tongue sensitivity. Hearing thresholds across all enrolled participants for each arm are shown in fig. S3. Treatment was selfadministered by participants at home using the device for a recommended 60 minutes per day with a minimum compliance criterion of 36 hours over the full treatment period. The device was returned at the 12-week visit and participants were assessed at three follow-up visits over 12 months.

As described in the previously published protocol paper (41), the primary endpoints comprised of: (i) within-arm changes in the THI and TFI outcome measures from baseline (average of scores from screening and enrollment visits) to end of treatment for participants who were 
treatment-compliant (i.e., a per-protocol analysis in which participants used the device for at least 36 hours during the intended 12-week period); and (ii) between-arm differences in the changes in THI and TFI outcome measures from baseline to end of treatment for the full cohort of participants (i.e., an intention-to-treat analysis where missing data points were estimated using a multiple imputation method). Both THI and TFI are questionnaires with global scores from 0 to 100 with a higher score indicating greater tinnitus symptom severity.

To initially visualize the therapeutic effects experienced by each participant, Fig. 3 displays a scatter plot for each treatment arm. The final THI or TFI score for each treatment-compliant participant is plotted against the baseline score. Most of the participants exhibited an improvement in THI or TFI during treatment, ranging between $74.7 \%$ and $88.8 \%$ across arms. There was also a trend towards greater improvement in THI or TFI for those who had worse tinnitus symptoms at baseline, with many participants achieving large improvements (e.g., a decrease in 20 or 30 points; fig. S4). These positive therapeutic effects were observed for all three stimulation settings.

For the primary endpoints of the study, Fig. 4 presents the averaged results across participants for the within-arm and between-arm comparisons in a forest plot format. All withinarm comparisons, for both THI and TFI, were highly significant based on paired two-tailed t-tests $(P<0.001)$. Within-arm primary endpoints were successfully achieved and statistically significant when accounting for multiple comparisons. Arms 1, 2 and 3 exhibited a decrease of 14.6, 14.5 and 13.5 points in THI, and 13.9, 13.8 and 13.2 points in TFI, respectively. In terms of Cohen's d effect sizes and $95 \%$ confidence intervals, Arms 1, 2 and 3 exhibited values of -0.92 [-1.14, -0.71$],-0.87$ $[-1.08,-0.67]$, and $-0.92[-1.16,-0.69]$ for THI, and -0.87 [-1.10, -0.65], $-0.77[-0.98-0.56]$, and $-0.78[-1.05,-0.51]$ for TFI, respectively. We did not observe a significant difference between arms during the treatment phase $(P>0.05$ for between-arm comparisons; ANCOVA: $\mathrm{F}(3,270)=58.45$, $P>0.05$; description and justification of statistical analyses are provided in the Materials and Methods).

\section{Long-term treatment outcomes}

The study was designed to track changes in tinnitus symptom severity at multiple time points during treatment and up to 12 months after the end of treatment. Figure 5 plots the mean change in THI and TFI relative to baseline value for each assessment visit for the three study arms. There were three interesting observations from the data presented in Fig. 5. First, all three stimulation settings resulted in rapid improvement within the first 6 weeks of treatment. Second, there were minimal improvements in the second 6 weeks of treatment, which may be attributed to habituation processes in the brain. Third, improvements were significantly sustained out to 12 months after treatment ( $P<0.05 ; 95 \%$ confidence interval does not cross the zero line), except for Arm 3. There was also a greater proportion of participants retaining an improvement after 12 months post-treatment for Arm 1 and Arm 2 compared to Arm 3 (fig. S5 and S6; though not significant based on Fisher's Exact Test, $P>0.05$ ), which corresponded to greater sustained effects for Arm 1 and Arm 2 compared to Arm 3 (significant differences between arms are listed in Fig. 5). These long-term findings suggest that bimodal neuromodulation utilizing higher frequency 
tones with synchronized or shorter delayed tongue stimulation could be more effective in delivering a sustained therapeutic benefit in a higher percentage of tinnitus patients compared to bimodal neuromodulation with low frequency tones and long delayed tongue stimulation. Since there were no apparent differences in outcomes between Arm 1 and Arm 2, the fixed tone-totongue mapping is not necessary to drive therapeutic effects. Arm 1 and 2 delivered a greater number of stimulus bursts per second than in Arm 3, which could have also contributed to the differences in long-term outcomes.

Previous studies have shown that many people with tinnitus have high frequency hearing loss and a dominant tinnitus pitch that falls within the compromised spectral region $(25,52,53)$. Presenting sounds with high frequency components and/or matched to the dominant tinnitus pitch have been proposed as effective methods for treating tinnitus, both for sound-only approaches and for bimodal neuromodulation (30,54-56). Alleviating the tinnitus percept has also been achieved through cochlear implant stimulation by re-activating compromised hearing pathways $(57,58)$. To further assess the possible contribution of high frequency tones in the long-term therapeutic effects shown in Fig. 5, we plotted the change in THI or TFI as a function of high frequency hearing loss (averaged across 4 to $8 \mathrm{kHz}$; fig. S7 and S8). If high frequency tones are critical, especially since many of our participants had high frequency hearing loss across arms, we would expect less therapeutic benefit for those with greater high frequency hearing loss, particularly for Arm 3 where PS3 consisted of only low frequency sounds $(\sim 100-500 \mathrm{~Hz})$. We did not observe any relationship between therapeutic effects and high frequency hearing loss, either at the end of treatment or at the 12-month post-treatment assessment. When plotting the change in THI or TFI as a function of hearing loss only for $8 \mathrm{kHz}$ (i.e., the band with the greatest high frequency hearing loss across participants; fig. S9 and S10), a trend emerged for Arm 3 at the end of treatment but it was not significant $(P>0.05)$ at the 12-month post-treatment assessment. These trends were weak with high variability; thus, the high frequency component of PS1 and PS2 could be contributing to therapeutic outcomes for those with high frequency hearing loss, but was not the main driver.

In terms of overall stimulation rate, there was no clear indication that the reduced number of stimulus bursts per second in PS3 compared to PS1 and PS2 affected overall efficacy (fig. S11). This finding is consistent with the observation that all arms exhibited rapid and equivalent reductions in THI and TFI within 6-weeks of treatment with minimal additional reductions during the second 6-week period, suggesting that participants using PS3 were receiving more than the minimum number of stimulus bursts for the intervention to be effective. However, from these data the contribution of stimulation rate on long-term outcomes cannot be completely ruled out, and should be further investigated in a follow-up study.

To summarize the overall short-term and long-term therapeutic benefits of bimodal neuromodulation, Figure 6 collapses the three study arms and pools data for all treatmentcompliant participants. Pooled THI and TFI scores are plotted for baseline versus final treatment assessment (Fig. 6, A and B) and baseline versus 12-month follow-up assessment (Fig. 6, C and D). These data provide evidence that, irrespective of stimulation parameters, the majority of treatment-compliant participants experienced an improvement in tinnitus symptoms after 12 weeks of treatment ( $>81 \%)$ and at 12 months post-treatment ( $>77 \%)$, based on a large sample size of $n \geq$ 
151. The remaining $\sim 20 \%$ of participants experienced no change or an increase in their tinnitus symptoms (Fig. 6 shows the extent of increase in THI or TFI for those individuals). Based on the data from Fig. 6, there was a decrease of 14.2 points (SD:13.4; Cohen's d: -0.91 [-1.04, -0.79]) at the end of treatment that was sustained to 12.7 points (SD: 16.2; Cohen's d: $-0.81[-1.00,-0.62]$ ) at 12 -months post-treatment for THI. There was also a decrease of 13.6 points (SD: 16.2; Cohen's d: $-0.81[-0.94,-0.67])$ at the end of treatment that was sustained to 14.5 points (SD: 18.6; Cohen's d: $-0.80[-0.99,-0.61])$ at 12 -months post-treatment for TFI. These results represent one of the largest and longest post-treatment datasets for a medical device study in the tinnitus field.

\section{Safety and acceptability of device}

A Medical Research Organization (NAMSA, Minneapolis, Minnesota, USA) guided and assisted the close-out process of our clinical trial, in addition to reviewing and categorizing safety data together with the Medical Review Board of the study. An adverse event (AE) was classified in line with MEDDEV 2.7/3 and defined as any untoward medical occurrence, unintended disease or injury, or any untoward clinical signs in a subject, user or other person whether or not related to the investigational medical device. A serious adverse event (SAE) was defined as an AE that led to death, injury or permanent impairment to a body structure or a body function; or led to a serious deterioration in health of the subject, that either resulted in: (i) a life-threatening illness or injury, (ii) a permanent impairment of a body structure or a body function, (iii) an in-patient hospitalization or prolongation of existing hospitalization, or (iv) a medical or surgical intervention to prevent life threatening illness.

There were no treatment-related SAEs in this study. Table 2A lists 155 AEs that were potentially related to the treatment. These AEs were categorized into three causality groups: (i) probably device related, (ii) possibly device related, and (iii) probably not device related; and further classified as moderate or negligible in terms of severity. 106 of the 155 AEs were expected AEs and were described in the clinical study protocol, the device labeling (User Manual) and informed consent documents. The most common expected AE was an increase in tinnitus symptoms, and was further subdivided into two categories: a dramatic increase in tinnitus symptoms or a subjective increase in tinnitus symptoms. A dramatic increase corresponded to a substantial increase in tinnitus that was noticeably bothersome to the participant. A subjective increase corresponded to a participant experiencing a change in their tinnitus, which was not considered dramatic, but could be occasionally or moderately bothersome or non-bothersome. Other expected AEs included discomfort or pain in the head, ear or mouth area, as well as discomfort or an ulceration within the oral cavity or on the tongue. There were also 17 unanticipated AEs (Table 2, A and B). Table 2A includes a separate category listed as an "event caused by patient conditions," which were AEs attributed to other health or personal conditions. Most of these were negligible AEs (24 out of 27) with three moderate cases (see table S2).

Comparing differences in each $\mathrm{AE}$ sub-category across treatment arms was not possible due to the low number of instances in each arm. However, there was a sufficient number of cases for subjective increase in tinnitus by pooling across causality groups (57 total cases), in which 
there was no significant difference among treatment arms (Arm 1:21, Arm 2: 17, Arm 3:19; $P=$ 0.920, Fisher's exact test).

In summing all cases for dramatic increase or subjective increase in tinnitus symptoms, there is a total of 64 AEs listed in Table 2a, which correspond to 52 unique individuals (i.e., a single participant could have more than one $\mathrm{AE}$ for increased tinnitus symptoms that occurred throughout the study). 47 of these 52 unique AEs happened during treatment, which is consistent with the number of participants who did not exhibit an improvement in THI or TFI at the end of treatment (36 for THI and 48 for TFI based on Fig. 6).

Overall, the treatment proved to be safe with no SAEs and a high satisfaction rate across a large cohort of participants. There were various AEs listed in Table 2 that may have caused discomfort to the participants, but the majority of the participants (273 out of 326) still used the device for at least the minimum compliance of 36 hours over the 12 -week treatment period. At the end of treatment, participants were asked, "overall, would you say you have benefitted from using this device?" Out of 272 responses, $66.5 \%$ indicated "Yes". They were also asked, "if you knew someone with tinnitus would you recommend they try this treatment?" Out of 270 responses, $77.8 \%$ indicated "Yes". Those who responded "Yes" to these two questions generally exhibited a greater reduction in tinnitus symptoms compared to those who responded "No" (see fig. S12 and S13). These high compliance and satisfaction rates, when compared to the reported AEs, support a strong benefit-to-risk profile for this medical device treatment for tinnitus.

\section{Discussion}

The main objective of this study was to build further safety and efficacy data, based on a previous pilot study in humans (31). This study was designed to explore the effects of different stimulation parameters on tinnitus symptoms in a broad tinnitus population and in specific subtypes of patients to determine if they are differentially responsive to certain stimulation settings. Overall, the study represents one of the largest medical device clinical trials in the tinnitus field, supporting the safety, patient tolerability and efficacy of bimodal neuromodulation for the treatment of tinnitus.

Out of 326 enrolled participants, $84 \%$ used the device at or above the minimum compliance criterion of 36 hours over the 12 -week treatment period. Of the treatment-compliant participants, 74.7-88.8\% demonstrated improvements after 12 weeks of treatment with an average decrease of 13.2 to 14.6 points for different arms in THI and TFI, corresponding to a large Cohen's $\mathrm{d}$ effect size ranging from -0.77 to -0.92 . The extent of improvement observed in this study is comparable to clinically meaningful effects reported in other well-designed clinical trials $(28,45,47,59)$. Currently, the only clinically validated and recommended treatment for tinnitus is cognitive behavioral therapy (CBT) $(28,59)$. A seminal randomized controlled trial reported improvements in THI of about 10 points after 8 months of a specialized CBT treatment (60). A recently published Cochrane review for CBT also highlighted an average improvement in THI of 10.9 points by the end of treatment (61). In our study, bimodal neuromodulation achieved 14.2 points mean improvement in THI within 6-12 weeks of self-administered treatment that could be sustained for 
12 months post-treatment at 12.7 points. To the best of our knowledge, no other tinnitus treatment has demonstrated such long-term post-treatment effects in a large-scale clinical trial.

In the follow-up phase of the study, we observed different trends that emerged between the three stimulation settings. The more synchronized settings (PS1 and PS2) exhibited sustained effects that were more consistent over time compared to that of PS3, suggesting that shorter interstimulus delays or synchronization between sound and tongue stimulation could be a key feature for driving long term plasticity. PS1 and PS2 consisted of higher frequency pure tones and a greater number of stimulus bursts per second (i.e., about 25 times greater) compared to the stimulus features in PS3, which may have also contributed to the long-term benefit observed.

Further support for the efficacy of bimodal neuromodulation was provided in a previous pilot study by Marks et al. (30) in 2018 that evaluated a device presenting sound combined with electrical somatosensory stimulation via the cheek or neck region. Twenty tinnitus participants were randomized (1:1) to a bimodal treatment group or an auditory-only control group, and underwent 30 minutes of daily treatment for 4 weeks. The bimodal stimulation group achieved significant improvements in tinnitus symptom severity (e.g., an average reduction of 6.3 points in TFI), while the auditory-only group did not achieve a noticeable improvement in symptoms. Therapeutic effects remained for about three weeks post-treatment. In our study, TFI decreased by 13.6 points with residual therapeutic effects that were sustained out to 12 months post-treatment at 14.5 points. There are several possible reasons for the differences in long-term effects. The Marks et al. (30) study used bimodal stimulation consisting of electrical stimulation of the cheek or neck, in contrast to stimulation of the tongue in our study. They also used different acoustic stimuli (i.e., a matched tinnitus spectrum for each participant) and a specific inter-stimulus timing of $5 \mathrm{~ms}$. We used a diverse range of acoustic stimuli (i.e., different frequency ranges) and delays across treatment arms yet still observed long-term benefits for all arms, suggesting that these stimulus features do not appear to be the main reason for the discrepancy. Treatment in the Marks et al. (30) study was only presented for 30 minutes per day for 4 weeks (a total of 14 hours of stimulation), whereas we used a treatment paradigm with a minimum of 36 hours of stimulation for 12 weeks. Based on the concept of paired plasticity $(7,8,17,20,23)$, repeated bimodal stimulation over a longer period of time may have driven greater and/or longer-lasting changes in the brain in our study. Together with our results, these observations suggest that a diverse range of acoustic and somatosensory stimulation patterns are effective in reducing tinnitus symptoms, but that a minimum duration or repetition of paired stimulation is required to achieve long-lasting therapeutic effects. Furthermore, long-term improvements in tinnitus remain robust to interstimulus delays between sound and somatosensory stimulation that vary on the order of tens of milliseconds but appear to diminish with delays exceeding hundreds of milliseconds.

Interestingly, the Marks et al. (30) study reported that repeated paired stimulation could drive continued improvements in tinnitus symptoms with each week of bimodal neuromodulation for their 4-week regimen. However, our study indicates that there may be a limit as to the degree of improvement achieved when using the same stimulation setting. During the second 6-weeks of stimulation, we did not observe as large of an improvement in tinnitus symptoms as occurred during the first 6-weeks of treatment. There may be habituation effects where the brain becomes 
less sensitive in response to repetitive stimuli over time $(22,62-65)$. One way to overcome habituation could be to change the stimulation setting over the course of a treatment regimen (e.g., after 6 weeks of treatment). This stimulus adjustment may potentially lead to greater improvements in tinnitus symptoms that could be sustained over a longer post-treatment period. We are currently engaged in another large-scale clinical trial evaluating the effects of varying bimodal stimulation parameters over time for tinnitus treatment (66).

One limitation in the study is that all three arms consisted of bimodal neuromodulation with supra-threshold intensities of sound and tongue stimulation that were required to maintain blinding, and they showed similar improvements in THI and TFI scores at the end of treatment. Based on the concept of paired plasticity, a significant difference would have been expected between the synchronized Arm 1 and less synchronized Arm 3. Although this between-arm endpoint was not achieved at the end of treatment, differences in THI and TFI scores emerged between arms during the post-treatment phase, suggesting that different stimulation settings can lead to different long-term treatment outcomes. Prolonged residual effects lasting 12 months would also not be expected for placebo effects. The bimodal stimulation paradigm used in Marks et al. (30) led to a significant improvement in tinnitus symptoms that returned back to baseline within three weeks. This intervention was believed to be a legitimate treatment by the tinnitus participants, demonstrating that simply informing participants that they are receiving an effective bimodal treatment does not lead to a 12-month residual effect. As described above, there appears to be a minimum duration of bimodal neuromodulation (>14-36 hours) required to achieve longterm effects, which can be further sustained by using shorter delays between sound and tongue stimulation $(<50-550 \mathrm{~ms})$. Therefore, future clinical studies can be designed to reveal differences in long-term outcomes between treatment arms that diverge toward opposite extremes of those two parameter dimensions.

\section{Materials and Methods}

\section{Study design}

We conducted a parallel-arm, double-blind, randomized, exploratory study investigating the safety and efficacy of three different stimulation settings. The study was performed at two different clinical sites. One site was the Wellcome Trust-HRB Clinical Research Facility at St. James's Hospital in Dublin, Ireland, and the second site was the Tinnituszentrum of the University Regensburg at the Bezirksklinikum Regensburg in Germany. The study protocol was independently reviewed and approved by the Research Ethics Committees of the Tallaght Hospital - St. James's Hospital (Protocol Number: 2016-03-List-11(3)) and by the University Hospital Regensburg (Protocol Number: 16-101-0186). A detailed description of the study design, protocol and planned analyses were previously published in a protocol paper (41) and the clinical trial is registered at clinicaltrials.gov (NCT02669069; (67)). Informed consent was obtained from each of the 698 participants at the screening visit. One candidate decided not to sign the consent form during the consent process and was not enrolled in the study. The candidate had completed three 
questionnaires (THI, State-Trait Anxiety Inventory, and patient history). This protocol deviation was documented and reported to the appropriate Research Ethics Committee.

A total of 698 candidates were clinically screened at the two clinic sites. Of those candidates, 326 tinnitus participants were enrolled in the study. About $80 \%$ of the participants were enrolled at the Ireland clinical site and the remaining $20 \%$ were enrolled at the Germany clinical site (Table 1). The screening visit included various tinnitus, hearing, oral cavity and health assessments conducted by designated audiologists, clinicians and researchers to identify participants that satisfied the inclusion and exclusion criteria described in the next section. Each participant was assigned a Unique Identifier Code (UIC) so that participant files and data were managed in a de-identified manner throughout the study. Only designated investigators had access to the keys to decrypt UIC codes. Eligible participants were randomized to one of three different treatment arms. All participants and participant-facing investigators were blinded to the treatment assignment for each participant. When participants returned for their enrollment visit, we performed several tinnitus assessments and health evaluations relevant for monitoring adverse events. Each participant was then fitted with a bimodal neuromodulation device and provided a training session on how to use the device. We recommended two daily 30-minute treatment sessions over a 12-week period. The two sessions could be performed consecutively or at different times during the day. If the participants used the device for at least 36 hours over the full treatment period, then they were considered compliant to treatment, as described in the published protocol paper (41). During the treatment period, the participants attended the clinic for interim assessments at a 6-week visit and at the end of treatment at a 12-week visit. Tinnitus assessments and health evaluations were repeated across all visits. Participants returned their devices at the 12 -week visit and were asked to return for three follow-up visits at week 18 (6-week follow-up), week 38 (6month follow-up) and week 64 (12-month follow-up) to assess long-term safety and efficacy of the treatment. If there were cases that required additional medical assistance during the study, referrals were made to an appropriate clinician (three cases described in table S3). The study timeline is depicted in Fig. 1B with the number of participants attending and completing assessments listed in Fig. 2 and Table 1.

For randomization of participants to the three different treatment arms (1:1:1), stratification was performed based on four binary categories using the method of minimization (49), as described in the published protocol paper (41). These categories included atonal tinnitus, hyperacusis, normal hearing and noise induced hearing loss. Atonal tinnitus refers to participants who did not have a dominant tonal tinnitus. Hyperacusis was defined as a sound level tolerance (or loudness discomfort level, LDL) that was less than $60 \mathrm{~dB}$ sensation level (SL) for a pure tone presented at $500 \mathrm{~Hz}$ in either ear (dB SL equals dB HL minus hearing threshold level of acoustic stimulus). Since we did not incorporate additional clinical criteria and questionnaire data relevant for classifying hyperacusis into the stratification process, this category should be interpreted more strictly as a sound sensitivity condition specific to $500 \mathrm{~Hz}$ rather than what is typically considered as hyperacusis. The word "hyperacusis" is still used throughout this paper to remain consistent with the previously published stratification terminology for the clinical study (41). This hyperacusis criterion of $\mathrm{LDL}<60 \mathrm{~dB}$ SL is also summarized in units of $\mathrm{dB}$ hearing level (HL) to 
show that consistent stratification occurred for both types of units in our study (table S4). Normal hearing was defined as having pure-tone thresholds of $\leq 20 \mathrm{~dB}$ HL in all audiometric test frequencies between $250 \mathrm{~Hz}$ and $8 \mathrm{kHz}$ in both ears. Noise induced hearing loss was defined as having a dip in pure tone hearing thresholds of $10 \mathrm{~dB}$ or more in any of the measurement frequency bands of 3,4 or $6 \mathrm{kHz}$ in either ear. The classification was performed manually by a trained audiometrist wherein patients with either symmetrical dips or asymmetrical dips (e.g., $10 \mathrm{~dB}$ on the low frequency side and $5 \mathrm{~dB}$ on the high frequency side) were included. In some cases, participants could have substantial presbycusis that altered the audiograms used for classification, in which case these participants were included in the noise induced hearing loss category if they self-reported that their tinnitus was caused by noise exposure.

The tinnitus treatment device is a proprietary CE-marked Class IIa medical device developed by Neuromod Devices (Dublin, Ireland). The device comprises bimodal auditory and tongue stimulation (Fig. 1a). The auditory stimulus includes sequences of tone bursts with a continuous structured wideband noise in the background that are presented to both ears. The tongue is stimulated electrically via a 32-electrode transmucosal array placed on the anterior dorsal surface of the tongue. Electrical stimulation is delivered in the form of biphasic, anodic-leading pulses between approximately 5 and $210 \mu$ s duration and with a fixed amplitude. Bimodal stimulation with supra-threshold intensities for both sound and tongue stimulation was used for all three study arms, which enabled participant blinding to their assigned treatment. Supra-threshold settings of bimodal neuromodulation also achieved significant improvements in tinnitus symptom severity in a previous pilot study (31). Schematics and details of the stimulation parameters for each arm is presented in table S1. Briefly, Arm 1 (PS1 setting) consisted of synchronized sound and tongue stimulation. A given pure tone ( $\sim 15 \mathrm{~ms}$ duration, $500-8000 \mathrm{~Hz}$, repetition period of $\sim 80 \mathrm{~ms})$ was presented at the same time that a train of pulses was presented to a specific location on the tongue $(\sim 12-15 \mathrm{~ms})$. For Arm 1, the stimulation location on the tongue array was fixed for a given pure tone frequency; this resulted in a fixed tone-to-tongue mapping. Note that the 32-electrode tongue array shown in Fig. 1a was divided into two halves (left and right), such that symmetrical locations on each side of the tongue were electrically stimulated as the corresponding pure tone was presented binaurally (table S1). Arm 2 (PS2 setting) used the same auditory stimulus as Arm 1, but had an inter-stimulus delay that varied in the range of 30-50 ms across each stimulus presentation. The tone-to-tongue mapping was also randomized across stimulus presentations. Arm 3 (PS3 setting) used lower frequency tones (100-500 Hz), longer varying inter-stimulus delays (550-950 ms) and a randomized tone-to-tongue mapping as in Arm 2. These longer delays also required a longer repetition period, which was set to $\sim 2 \mathrm{~s}$ for PS3. Arm 3 with PS3 was designed to consist of a much less synchronized and less coordinated setting compared to PS1 used in Arm 1. Based on the concept of paired stimulation described in the Introduction (17, 20, 23, 30, 68-70), the greatest treatment differences would be expected between Arm 1 and Arm 3. Greater synchrony or more coordinated activation of bimodal neuromodulation should drive stronger therapeutic plasticity within brain regions related to tinnitus.

Sound volume range and electrical tongue stimulation intensities were customized to each participant's sensation thresholds. The participant's pure-tone audiometric thresholds $(250 \mathrm{~Hz}$ to 
$8 \mathrm{kHz}$ ) were measured at the screening visit (fig. S3) and subsequently used to configure the intensity of the auditory stimuli to $\sim 10$ decibels in sensation level (dB SL) above their hearing thresholds at each tone frequency. The participant could adjust the default auditory stimulus intensity between $-12 \mathrm{~dB}$ and $+12 \mathrm{~dB}$ during treatment using volume buttons on the controller. For safety reasons, the upper stimulus intensity was limited for participants with profound hearing loss (up to $\sim 90 \mathrm{dBA}$ ). Electrical stimulation intensity (i.e., via changes in pulse duration for a fixed amplitude) was configured for each participant by adjusting the intensity from sub-threshold to supra-threshold sensations to a comfortable intensity across different electrodes, which was when the participant could feel sensations on the tongue but below an intensity that was bothersome or painful. This comfortable intensity was used as the calibrated setting, and the participant could adjust the electrical stimulation intensity down to $60 \%$ and up to $160 \%$ of this calibrated setting using buttons on the controller. The treatment device reverted to the default intensities at the start of each new treatment session.

\section{Participants}

The study recruited participants with chronic subjective tinnitus, which is a phantom auditory percept that is attributable to abnormal firing patterns in the brain (25). Subjective tinnitus differs from objective tinnitus, which is typically associated with sounds generated from vasculature or pulsation anomalies, abnormal muscle contractions or head/jaw movements (25). Participants in the age range of 18 and 70 with a tinnitus duration between 3 months and 5 years were included in the study. We recruited participants with THI scores of 28 to 76 points (44). We considered a score less than 28 as being sufficiently mild as to be less amenable to therapeutic intervention. A score greater than 76 is classified as catastrophic (44) and typically associated with other severe comorbidities including depression, anxiety and suicidal tendencies; these participants were not included due to the challenges in managing these complex comorbidities in a large-scale clinical trial. Only participants with a Minimum Masking Level (MML) between 20 and $80 \mathrm{~dB}$ HL were included in the study. MML was determined by presenting a wide-band noise at increasing intensities until the sound masked the participant's tinnitus. Additional inclusion criteria required that the participant was able to read and understand English at the Ireland site or read and understand German at the Germany site, was willing and able to provide informed consent, and was willing to commit to the full duration of the study.

Candidates were excluded if they had objective tinnitus or somatic tinnitus caused by a head or neck injury, or if their tinnitus was comorbid with a neurological condition as assessed by an audiologist or clinician. Conductive hearing loss demonstrated by abnormal otoscopy or abnormal tympanometry was an exclusion criterion. Substantial sensorineural hearing loss either unilaterally or bilaterally was also an exclusion criterion, since the headphones had an upper volume limit. Specifically, those candidates having $>40 \mathrm{~dB}$ HL in at least one measurement frequency in the range of $250 \mathrm{~Hz}$ to $1 \mathrm{kHz}$ or $>80 \mathrm{~dB}$ HL in at least one measurement frequency in the range of 2 to $8 \mathrm{kHz}$ were excluded. These hearing loss criteria corresponded to $10.2 \%$ or $5.3 \%$ of the screened individuals (out of 698 ) who were excluded from the study, respectively. Further exclusions included candidates with: a hearing aid used within 90 days prior to eligibility 
assessment, any type of electro-active implantable device (e.g., vagal nerve stimulator, cochlear implant or a cardiac pacemaker), a loudness discomfort level of $<30 \mathrm{~dB} \mathrm{SL}$ at $500 \mathrm{~Hz}$ for either ear, a temporomandibular joint disorder, anxiety determined by a score $>120$ out of 160 on the state-trait anxiety inventory (STAI) $(71,72)$, or moderate to severe dementia as indicated by a score $<20$ on the mini-mental state examination (MMSE) (73). A final set of exclusion criteria based on a medical history taken at the screening assessment were: Meniere's disease, oral piercings, pregnancy, involvement in medico-legal cases, history of auditory hallucinations, current prescription of a drug for a central nervous system pathology (e.g., epilepsy), and previous use of a Neuromod Devices' product.

\section{Primary endpoints}

The THI $(44,45)$ and TFI $(47)$ are clinical outcome measures commonly used to assess tinnitus symptom severity (74). These outcome measures have been used across multiple studies that have supported clinical guidelines for tinnitus interventions $(28,61)$. The only clinically recommended treatment for tinnitus, $\mathrm{CBT}$, has also leveraged these multi-item questionnaires for evidence that it is an effective tinnitus intervention (61). The THI predominantly assesses the emotional and functional impact of tinnitus, in which 25 items are scored $4 / 2 / 0$ on a categorical scale corresponding to yes/sometimes/no. The global score of the THI (i.e., sum of points across all 25 items) has a value from 0 to 100 , with a higher score indicating a greater negative impact of tinnitus. The TFI assesses a range of tinnitus-related functional complaints experienced over the week prior to assessment. Each of the 25 items is assessed on an 11-point Likert scale, and the sum of the scores is normalized to give a global score from 0 to 100 , with a higher score indicating a greater negative impact of tinnitus.

\section{Changes and deviations to the trial design}

The target sample size assumed 91 participants in each treatment arm at the 12-week endpoint, with the design accounting for an expected drop-out rate of $20 \%$ for a total of 342 participants. The achieved retention rate was better than predicted and so recruitment was stopped at 326 participants based on the accumulating data.

Of the 326 enrolled participants, 34 of them did not meet the eligibility criteria and are considered deviations to the protocol. Ten had a tinnitus duration greater than five years, three had a THI score less than 28 points, 16 scored below 20 points on the MMSE, two had an MML of greater than $80 \mathrm{~dB} \mathrm{HL}$, two had previously been involved in a medical legal case, and one indicated they had a neurological disorder. In addition to these 34 participants, there were 29 who had at least one missing entry for their eligibility assessment such that it was not possible to confirm if there was a deviation to the inclusion and exclusion criteria. Analyses were conducted with and without these participants. The sensitivity analysis demonstrated no significant effect on the primary endpoint findings $(P>0.05)$; thus, all of these participants were included in the analyses presented in this paper. 
During the database validation process, there were 10 participants whose audiogram data were identified as being incorrectly inputted into the database at the screening visit, in which their hearing threshold values were supposed to be higher than what was inputted into the database (i.e., by 5 to $25 \mathrm{~dB}$ across one to five frequencies in the range of 500 to $8000 \mathrm{~Hz}$ ). In these cases, the sound volume was not set to be loud enough for some frequencies. Analyses were conducted with and without these participants. The sensitivity analysis demonstrated no significant effect on the primary endpoint findings $(P>0.05)$; thus, all of these participants were also included in the analyses presented in this paper.

\section{Statistical analyses}

The primary endpoints of the study were designed together with statistical experts from NAMSA Medical Research Organization (Minneapolis, Minnesota, USA), and are described in a previously published protocol paper (41). These endpoints included within-arm changes from baseline to end of treatment at 12-weeks (final) for THI and TFI, and between-arm differences in changes from baseline to final for THI and TFI. Baseline was calculated as the average of the THI or TFI scores at screening and enrollment. The within-arm analyses were based on a per-protocol estimand and tested with paired two-tailed t-tests, where normality assumption was confirmed with the Shapiro-Wilk test. Within-arm effect sizes reported in the paper were based on Cohen's $d$ and are calculated as the mean score at end of treatment minus the mean score at baseline, divided by the pooled SD for the two scores. The between-arm analyses were based on an intention-to-treat estimand with multiple imputation and tested with multiple regression using baseline scores as a covariate, where normality and equal variance assumptions were confirmed with the Shapiro-Wilk test and Bartlett's test, respectively. An ANCOVA was also performed across arms. Missing data were handled by using a Markov chain Monte Carlo multiple imputation method $(75,76)$ that filled in the missing predictor variables for 10 multiple imputed datasets. This procedure was followed by a second imputation process that estimated the final outcome variable within each imputed dataset. The imputation was based on a regression model with predictor variables of age, gender, tinnitus tonality, degree of somatic modulation, duration of tinnitus, treatment arm, treatment compliance, high frequency hearing loss, degree of sound intolerance, and baseline and post-treatment THI, TFI and MML scores. Inferences for the between-arm endpoints were evaluated on each imputed data set and the results combined to yield the estimates, confidence intervals and associated significance values. The use of per-protocol estimand for within-arm analyses ensured that the changes in outcome measures within each treatment arm were reflective of daily use scenarios where the participants use the treatment as directed; and thus, included only the participants who were compliant to treatment. The threshold for inclusion in the per-protocol analysis was set at a minimum compliance threshold of 36 hours across the intended 12-week treatment period, as described in the protocol paper (41). We were able to track daily usage and stimulus adjustments that were logged automatically by the device, such as the time and date when the device was in use, the duration of electrode contact with the tongue, and the intensities of both the auditory and tongue stimuli for each treatment session. 
The study was conducted at two clinical sites with the intention of pooling data from both sites for the primary endpoint inferences. A test for site interaction on the primary between-arm endpoints (comparing mean change in scores from baseline to final assessments) was conducted and found to be not statistically significant $(P>0.05$ for both THI and TFI, using linear regression with an interaction term of clinical site and treatment arm, and with baseline score as a covariate). Therefore, the data from each clinical site was combined as intended in the study design to perform the analyses presented in this paper. Figures S14 and S15 further show scatter plots for the baseline and final THI and TFI scores, respectively, for each treatment arm and clinical site.

\section{Supplementary Materials}

Fig. S1. Compliance and satisfaction rates in using treatment device.

Fig. S2. Histogram of time (in weeks) when participants attended each visit relative to enrollment.

Fig. S3. Hearing thresholds for treatment-compliant participants.

Fig. S4. Scatter plots for change in THI or TFI score.

Fig. S5. Scatter plots for THI scores at baseline versus 12-month post-treatment assessment for each arm.

Fig. S6. Scatter plots for TFI scores at baseline versus 12-month post-treatment assessment for each arm.

Fig. S7. Scatter plots for change in THI or TFI score (from baseline to end of 12-week treatment) versus high frequency hearing loss (4 to $8 \mathrm{kHz})$ for each arm.

Fig. S8. Scatter plots for change in THI or TFI score (from baseline to 12-month post-treatment assessment) versus high frequency hearing loss (4 to $8 \mathrm{kHz}$ ) for each arm.

Fig. S9. Scatter plots for change in THI or TFI score (from baseline to end of 12-week treatment) versus $8 \mathrm{kHz}$ hearing loss for each arm.

Fig. S10. Scatter plots for change in THI or TFI score (from baseline to 12-month post-treatment assessment) versus $8 \mathrm{kHz}$ hearing loss for each arm.

Fig. S11. Scatter plots for change in THI or TFI score versus duration of device usage.

Fig. S12. Changes in tinnitus symptoms based on responses to first satisfaction question.

Fig. S13. Changes in tinnitus symptoms based on responses to second satisfaction question.

Fig. S14. Scatter plots for THI scores for each treatment-compliant individual at baseline versus end of treatment (12-week "final" visit) for each arm.

Fig. S15. Scatter plots for TFI scores for each treatment-compliant individual at baseline versus end of treatment (12-week "final" visit) for each arm.

Table S1. Schematics and description of stimulation setting used in each arm. 
Table S2. Adverse events attributed to patient conditions from Table 2.

Table S3. Patient referrals for adverse events.

Table S4. Stratification category of hyperacusis in Table 1 calculated in units of dB HL.

\section{References}

1. S. Frere, I. Slutsky, Alzheimer's Disease: From Firing Instability to Homeostasis Network Collapse. Neuron 97, 32-58 (2018).

2. J. A. Kaltenbach, Tinnitus: Models and mechanisms. Hear Res 276, 52-60 (2011).

3. R. Schaette, R. Kempter, Development of tinnitus-related neuronal hyperactivity through homeostatic plasticity after hearing loss: a computational model. Eur J Neurosci $\mathbf{2 3}, 3124-$ 3138 (2006).

4. A. Maffei, A. Fontanini, Network homeostasis: a matter of coordination. Curr Opin Neurobiol 19, 168-173 (2009).

5. T. Wise, L. Marwood, A. M. Perkins, A. Herane-Vives, R. Joules, D. J. Lythgoe, W. M. Luh, S. C. R. Williams, A. H. Young, A. J. Cleare, D. Arnone, Instability of default mode network connectivity in major depression: a two-sample confirmation study. Transl Psychiatry 7, e1105 (2017).

6. M. D. Johnson, H. H. Lim, T. I. Netoff, A. T. Connolly, N. Johnson, A. Roy, A. Holt, K. O. Lim, J. R. Carey, J. L. Vitek, B. He, Neuromodulation for brain disorders: challenges and opportunities. IEEE Trans Biomed Eng 60, 610-624 (2013).

7. A. V. Sathappan, B. M. Luber, S. H. Lisanby, The Dynamic Duo: Combining noninvasive brain stimulation with cognitive interventions. Prog Neuropsychopharmacol Biol Psychiatry 89, 347-360 (2019).

8. M. Bikson, A. R. Brunoni, L. E. Charvet, V. P. Clark, L. G. Cohen, Z. D. Deng, J. Dmochowski, D. J. Edwards, F. Frohlich, E. S. Kappenman, K. O. Lim, C. Loo, A. Mantovani, D. P. McMullen, L. C. Parra, M. Pearson, J. D. Richardson, J. M. Rumsey, P. Sehatpour, D. Sommers, G. Unal, E. M. Wassermann, A. J. Woods, S. H. Lisanby, Rigor and reproducibility in research with transcranial electrical stimulation: An NIMH-sponsored workshop. Brain Stimul 11, 465-480 (2018).

9. S. Vanneste, D. De Ridder, Noninvasive and invasive neuromodulation for the treatment of tinnitus: an overview. Neuromodulation 15, 350-360 (2012).

10. J. C. Horvath, U. Najib, D. Press, in Neuromethods, A. Rotenberg, J. C. Horvath, A. Pascual-Leone, Eds. (Springer, New York, 2014), vol. 89, chap. 12, pp. 235-257.

11. H. Yuan, S. D. Silberstein, Vagus Nerve and Vagus Nerve Stimulation, a Comprehensive Review: Part III. Headache 56, 479-490 (2016). 
12. H. Yuan, S. D. Silberstein, Vagus Nerve and Vagus Nerve Stimulation, a Comprehensive Review: Part II. Headache 56, 259-266 (2016).

13. A. M. Boasso, H. Mortimore, R. Silva, L. Aven, W. J. Tyler, Transdermal electrical neuromodulation of the trigeminal sensory nuclear complex improves sleep quality and mood. bioRxiv, 043901 (2016).

14. I. A. Cook, M. Abrams, A. F. Leuchter, Trigeminal Nerve Stimulation for Comorbid Posttraumatic Stress Disorder and Major Depressive Disorder. Neuromodulation 19, 299305 (2016).

15. C. M. DeGiorgio, J. Soss, I. A. Cook, D. Markovic, J. Gornbein, D. Murray, S. Oviedo, S. Gordon, G. Corralle-Leyva, C. P. Kealey, C. N. Heck, Randomized controlled trial of trigeminal nerve stimulation for drug-resistant epilepsy. Neurology 80, 786-791 (2013).

16. D. E. Chou, M. Shnayderman Yugrakh, D. Winegarner, V. Rowe, D. Kuruvilla, J. Schoenen, Acute migraine therapy with external trigeminal neurostimulation (ACME): A randomized controlled trial. Cephalalgia 39, 3-14 (2019).

17. C. D. Markovitz, B. T. Smith, C. D. Gloeckner, H. H. Lim, Investigating a new neuromodulation treatment for brain disorders using synchronized activation of multimodal pathways. Sci Rep 5, 9462 (2015).

18. T. M. Nienow, A. W. MacDonald, 3rd, K. O. Lim, TDCS produces incremental gain when combined with working memory training in patients with schizophrenia: A proof of concept pilot study. Schizophr Res 172, 218-219 (2016).

19. C. S. Gilmore, P. J. Dickmann, B. G. Nelson, G. J. Lamberty, K. O. Lim, Transcranial Direct Current Stimulation (tDCS) paired with a decision-making task reduces risk-taking in a clinically impulsive sample. Brain Stimul 11, 302-309 (2018).

20. N. D. Engineer, J. R. Riley, J. D. Seale, W. A. Vrana, J. A. Shetake, S. P. Sudanagunta, M. S. Borland, M. P. Kilgard, Reversing pathological neural activity using targeted plasticity. Nature 470, 101-104 (2011).

21. E. C. Meyers, N. Kasliwal, B. R. Solorzano, E. Lai, G. Bendale, A. Berry, P. D. Ganzer, M. Romero-Ortega, R. L. Rennaker, 2nd, M. P. Kilgard, S. A. Hays, Enhancing plasticity in central networks improves motor and sensory recovery after nerve damage. Nat Commun 10, 5782 (2019).

22. R. Markewitz, S. Engel, B. Langguth, M. Schecklmann, Effects of Acoustic Paired Associative Stimulation on Late Auditory Evoked Potentials. Brain Topogr 32, 343-353 (2019).

23. A. Guerra, V. Lopez-Alonso, B. Cheeran, A. Suppa, Solutions for managing variability in non-invasive brain stimulation studies. Neurosci Lett 719, 133332 (2020).

24. A. J. Heller, Classification and epidemiology of tinnitus. Otolaryngol Clin North Am 36, 239-248 (2003). 
25. A. R. Moller, B. Langguth, D. De Ridder, T. Kleinjung, Eds., Textbook of Tinnitus, (Springer Science+Business Media, LLC New York, 2011), pp. 1-816.

26. A. McCormack, M. Edmondson-Jones, S. Somerset, D. Hall, A systematic review of the reporting of tinnitus prevalence and severity. Hear Res 337, 70-79 (2016).

27. D. Baguley, D. McFerran, D. Hall, Tinnitus. Lancet 382, 1600-1607 (2013).

28. D. E. Tunkel, C. A. Bauer, G. H. Sun, R. M. Rosenfeld, S. S. Chandrasekhar, E. R. Cunningham, Jr., S. M. Archer, B. W. Blakley, J. M. Carter, E. C. Granieri, J. A. Henry, D. Hollingsworth, F. A. Khan, S. Mitchell, A. Monfared, C. W. Newman, F. S. Omole, C. D. Phillips, S. K. Robinson, M. B. Taw, R. S. Tyler, R. Waguespack, E. J. Whamond, Clinical practice guideline: tinnitus. Otolaryngol Head Neck Surg 151, S1-S40 (2014).

29. R. F. F. Cima, B. Mazurek, H. Haider, D. Kikidis, A. Lapira, A. Norena, D. J. Hoare, A multidisciplinary European guideline for tinnitus: diagnostics, assessment, and treatment. HNO 67, 10-42 (2019).

30. K. L. Marks, D. T. Martel, C. Wu, G. J. Basura, L. E. Roberts, K. C. Schvartz-Leyzac, S. E. Shore, Auditory-somatosensory bimodal stimulation desynchronizes brain circuitry to reduce tinnitus in guinea pigs and humans. Sci Transl Med 10, eaal3175 (2018).

31. C. Hamilton, S. D'Arcy, B. A. Pearlmutter, G. Crispino, E. C. Lalor, B. J. Conlon, An Investigation of Feasibility and Safety of Bi-Modal Stimulation for the Treatment of Tinnitus: An Open-Label Pilot Study. Neuromodulation 19, 832-837 (2016).

32. V. De Cicco, M. P. Tramonti Fantozzi, E. Cataldo, M. Barresi, L. Bruschini, U. Faraguna, D. Manzoni, Trigeminal, Visceral and Vestibular Inputs May Improve Cognitive Functions by Acting through the Locus Coeruleus and the Ascending Reticular Activating System: A New Hypothesis. Front Neuroanat 11, 130 (2017).

33. L. M. Aitkin, C. E. Kenyon, P. Philpott, The representation of the auditory and somatosensory systems in the external nucleus of the cat inferior colliculus. J Comp Neurol 196, 25-40 (1981).

34. B. R. Schofield, S. D. Motts, J. G. Mellott, Cholinergic cells of the pontomesencephalic tegmentum: connections with auditory structures from cochlear nucleus to cortex. Hear Res 279, 85-95 (2011).

35. S. Hormigo, A. Horta Junior Jde, R. Gomez-Nieto, D. E. Lopez, The selective neurotoxin DSP-4 impairs the noradrenergic projections from the locus coeruleus to the inferior colliculus in rats. Front Neural Circuits 6, 41 (2012).

36. L. M. Hurley, M. R. Sullivan, From behavioral context to receptors: serotonergic modulatory pathways in the IC. Front Neural Circuits 6, 58 (2012).

37. E. M. Rouiller, J. P. Hornung, F. De Ribaupierre, Extrathalamic ascending projections to physiologically identified fields of the cat auditory cortex. Hear Res 40, 233-246 (1989). 
38. K. Itoh, H. Kamiya, A. Mitani, Y. Yasui, M. Takada, N. Mizuno, Direct projections from the dorsal column nuclei and the spinal trigeminal nuclei to the cochlear nuclei in the cat. Brain Res 400, 145-150 (1987).

39. H. Li, N. Mizuno, Single neurons in the spinal trigeminal and dorsal column nuclei project to both the cochlear nucleus and the inferior colliculus by way of axon collaterals: a fluorescent retrograde double-labeling study in the rat. Neurosci Res 29, 135-142 (1997).

40. S. S. Yeo, P. H. Chang, S. H. Jang, The ascending reticular activating system from pontine reticular formation to the thalamus in the human brain. Front Hum Neurosci 7, 416 (2013).

41. S. D'Arcy, C. Hamilton, S. Hughes, D. A. Hall, S. Vanneste, B. Langguth, B. Conlon, Bimodal stimulation in the treatment of tinnitus: a study protocol for an exploratory trial to optimise stimulation parameters and patient subtyping. BMJ Open 7, e018465 (2017).

42. S. D. Koehler, S. E. Shore, Stimulus timing-dependent plasticity in dorsal cochlear nucleus is altered in tinnitus. $J$ Neurosci 33, 19647-19656 (2013).

43. C. D. Gloeckner, C. D. Markovitz, B. J. Smith, R. J. Hughes, H. H. Lim, Multimodal Synchronization Therapy: Exploring the effects of pinna stimulation. 9th International TRI Tinnitus Conference 9, 87-88 (2015).

44. C. W. Newman, G. P. Jacobson, J. B. Spitzer, Development of the Tinnitus Handicap Inventory. Arch Otolaryngol Head Neck Surg 122, 143-148 (1996).

45. F. Zeman, M. Koller, R. Figueiredo, A. Aazevedo, M. Rates, C. Coelho, T. Kleinjung, D. de Ridder, B. Langguth, M. Landgrebe, Tinnitus handicap inventory for evaluating treatment effects: which changes are clinically relevant? Otolaryngol Head Neck Surg 145, 282-287 (2011).

46. D. A. Hall, H. Haider, A. J. Szczepek, P. Lau, S. Rabau, J. Jones-Diette, A. Londero, N. K. Edvall, C. R. Cederroth, M. Mielczarek, T. Fuller, A. Batuecas-Caletrio, P. Brueggemen, D. M. Thompson, A. Norena, R. F. Cima, R. L. Mehta, B. Mazurek, Systematic review of outcome domains and instruments used in clinical trials of tinnitus treatments in adults. Trials 17, 270 (2016).

47. M. B. Meikle, J. A. Henry, S. E. Griest, B. J. Stewart, H. B. Abrams, R. McArdle, P. J. Myers, C. W. Newman, S. Sandridge, D. C. Turk, R. L. Folmer, E. J. Frederick, J. W. House, G. P. Jacobson, S. E. Kinney, W. H. Martin, S. M. Nagler, G. E. Reich, G.

Searchfield, R. Sweetow, J. A. Vernon, The tinnitus functional index: development of a new clinical measure for chronic, intrusive tinnitus. Ear Hear 33, 153-176 (2012).

48. V. A. Sanchez, D. A. Hall, B. Millar, C. D. Escabi, A. Sharman, J. Watson, S. Thasma, P. Harris, Recruiting ENT and Audiology patients into pharmaceutical trials: evaluating the multi-centre experience in the UK and USA. Int J Audiol 57, S55-S66 (2018).

49. S. J. Pocock, R. Simon, Sequential treatment assignment with balancing for prognostic factors in the controlled clinical trial. Biometrics 31, 103-115 (1975). 
50. I. P. Pavlov, Conditional reflexes: an investigation of the physiological activity of the cerebral cortex. (Oxford University Press, London, 1927).

51. D. O. Hebb, The organization of behavior. A neuropsychological theory. (John Wiley \& Sons, Inc., New York, 1949).

52. M. Sereda, D. A. Hall, D. J. Bosnyak, M. Edmondson-Jones, L. E. Roberts, P. Adjamian, A. R. Palmer, Re-examining the relationship between audiometric profile and tinnitus pitch. Int J Audiol 50, 303-312 (2011).

53. M. Sereda, M. Edmondson-Jones, D. A. Hall, Relationship between tinnitus pitch and edge of hearing loss in individuals with a narrow tinnitus bandwidth. Int J Audiol 54, 249-256 (2015).

54. K. M. Reavis, V. S. Rothholtz, Q. Tang, J. A. Carroll, H. Djalilian, F. G. Zeng, Temporary suppression of tinnitus by modulated sounds. J Assoc Res Otolaryngol 13, 561-571 (2012).

55. E. A. Koops, R. J. Renken, C. P. Lanting, P. van Dijk, Cortical Tonotopic Map Changes in Humans Are Larger in Hearing Loss Than in Additional Tinnitus. J Neurosci 40, 3178-3185 (2020).

56. A. J. Norena, An integrative model of tinnitus based on a central gain controlling neural sensitivity. Neurosci Biobehav Rev 35, 1089-1109 (2011).

57. A. K. Punte, K. Vermeire, A. Hofkens, M. De Bodt, D. De Ridder, P. Van de Heyning, Cochlear implantation as a durable tinnitus treatment in single-sided deafness. Cochlear Implants Int 12 Suppl 1, S26-29 (2011).

58. A. Perreau, R. Tyler, P. C. Mancini, Programming a Cochlear Implant for Tinnitus Suppression. J Am Acad Audiol 31, 302-308 (2020).

59. T. E. Fuller, H. F. Haider, D. Kikidis, A. Lapira, B. Mazurek, A. Norena, S. Rabau, R. Lardinois, C. R. Cederroth, N. K. Edvall, P. G. Brueggemann, S. N. Rosing, A. Kapandais, D. Lungaard, D. J. Hoare, R. F. Cima, Different Teams, Same Conclusions? A Systematic Review of Existing Clinical Guidelines for the Assessment and Treatment of Tinnitus in Adults. Front Psychol 8, 206 (2017).

60. R. F. Cima, I. H. Maes, M. A. Joore, D. J. Scheyen, A. El Refaie, D. M. Baguley, L. J. Anteunis, G. J. van Breukelen, J. W. Vlaeyen, Specialised treatment based on cognitive behaviour therapy versus usual care for tinnitus: a randomised controlled trial. Lancet 379, 1951-1959 (2012).

61. T. Fuller, R. Cima, B. Langguth, B. Mazurek, J. W. Vlaeyen, D. J. Hoare, Cognitive behavioural therapy for tinnitus. Cochrane Database Syst Rev 1, CD012614 (2020).

62. G. Berding, F. Wilke, T. Rode, C. Haense, G. Joseph, G. J. Meyer, M. Mamach, M. Lenarz, L. Geworski, F. M. Bengel, T. Lenarz, H. H. Lim, Positron Emission Tomography Imaging Reveals Auditory and Frontal Cortical Regions Involved with Speech Perception and Loudness Adaptation. PLoS One 10, e0128743 (2015). 
63. D. De Ridder, S. Vanneste, E. van der Loo, M. Plazier, T. Menovsky, P. van de Heyning, Burst stimulation of the auditory cortex: a new form of neurostimulation for noise-like tinnitus suppression. J Neurosurg 112, 1289-1294 (2010).

64. A. Fasano, R. C. Helmich, Tremor habituation to deep brain stimulation: Underlying mechanisms and solutions. Mov Disord 34, 1761-1773 (2019).

65. M. T. Barbe, L. Liebhart, M. Runge, K. A. Pauls, L. Wojtecki, A. Schnitzler, N. Allert, G. R. Fink, V. Sturm, M. Maarouf, L. Timmermann, Deep brain stimulation in the nucleus ventralis intermedius in patients with essential tremor: habituation of tremor suppression. $J$ Neurol 258, 434-439 (2011).

66. B. Conlon, C. Hamilton, S. Hughes, E. Meade, D. A. Hall, S. Vanneste, B. Langguth, H. H. Lim, Noninvasive Bimodal Neuromodulation for the Treatment of Tinnitus: Protocol for a Second Large-Scale Double-Blind Randomized Clinical Trial to Optimize Stimulation Parameters. JMIR Res Protoc 8, e13176 (2019).

67. Clinicaltrials.gov, Treatment Evaluation of Neuromodulation for Tinnitus (NCT02669069). https://clinicaltrials.gov/ct2/show/NCT02669069.

68. Y. Xiong, Y. Zhang, J. Yan, The neurobiology of sound-specific auditory plasticity: a core neural circuit. Neurosci Biobehav Rev 33, 1178-1184 (2009).

69. S. A. Hays, Enhancing Rehabilitative Therapies with Vagus Nerve Stimulation. Neurotherapeutics 13, 382-394 (2016).

70. N. M. Weinberger, Specific long-term memory traces in primary auditory cortex. Nat Rev Neurosci 5, 279-290 (2004).

71. C. D. Spielberger, R. L. Gorsuch, R. Lushene, P. Vagg, G. Jacobs, Consulting Psychologists Press; Palo Alto, CA: 1983. Manual for the state-trait anxiety inventory, (1983).

72. K. Kvaal, I. Ulstein, I. H. Nordhus, K. Engedal, The Spielberger state-trait anxiety inventory (STAI): the state scale in detecting mental disorders in geriatric patients. Int $J$ Geriatric Psych 20, 629-634 (2005).

73. M. F. Folstein, S. E. Folstein, P. R. McHugh, "Mini-mental state": a practical method for grading the cognitive state of patients for the clinician. J Psych Res 12, 189-198 (1975).

74. D. A. Hall, H. Haider, A. J. Szczepek, P. Lau, S. Rabau, J. Jones-Diette, A. Londero, N. K. Edvall, C. R. Cederroth, M. Mielczarek, Systematic review of outcome domains and instruments used in clinical trials of tinnitus treatments in adults. Trials 17, 270 (2016).

75. D. B. Rubin, Multiple imputation for nonresponse in surveys. (John Wiley \& Sons, 2004), vol. 81.

76. J. L. Schafer, Analysis of incomplete multivariate data. (Chapman and Hall/CRC, 1997).

77. G. D. Ruxton, The unequal variance t-test is an underused alternative to the Student's t-test and the Mann-Whitney U test. Behav Ecol 17, 688-690 (2006). 


\section{Acknowledgments}

We thank M. Foster, A. M. Leonard, G. Kelly, A. Nolan, D. Nolan, A. Fitzpatrick, M. Holton, M. Lynch, T. Kessie, E. Cremen, M. Craig, G. Foley, and D. Reidy and her team of Clinical Research Nurses from the Ireland clinical site; as well as J. Rosenberger, A. Feyer, G. Charlot, F. Brandt and S. Staudinger from the Germany clinical site for performing evaluations with the participants, organization/execution of the study, and/or data collection during the study. We thank M. Foster and A. Prokopiou for assisting with some of the data analysis and in preparing figures or tables for the publication. We also thank NAMSA Medical Research Organization (Minneapolis, Minnesota, USA) for expert guidance and support in validating and closing-out the database, as well as in reviewing, analyzing and summarizing the safety and efficacy data. Funding: This work was sponsored by Neuromod Devices (Dublin, Ireland). Author contributions: $\mathrm{SD}, \mathrm{CH}, \mathrm{SH}, \mathrm{BC}, \mathrm{BL}, \mathrm{DAH}, \mathrm{SV}$ helped design and set up the study. $\mathrm{BC}, \mathrm{BL}, \mathrm{CH}$, COC, MS, TS, SD were involved with recruitment and screening of subjects. SH generated the random allocation sequence and assigned participants to each arm. $\mathrm{CH}, \mathrm{COC}, \mathrm{MS}, \mathrm{BL}$ coordinated and/or performed the study assessments and documentation tasks. SH programmed the devices and managed technical support. $\mathrm{BC}, \mathrm{BL}, \mathrm{CH}, \mathrm{TS}, \mathrm{SH}, \mathrm{EM}, \mathrm{HHL}$ evaluated and helped to categorize the adverse events. HHL, EM, SH were involved with overseeing and closing out the study. HHL, EM, SH, BC, CH, BL, DA, SV, MS, SLL, TS analyzed and/or interpreted the data. HHL drafted the manuscript with contributions from the other authors. Competing interests: $\mathrm{BC}$, $\mathrm{CH}, \mathrm{SH}, \mathrm{EM}, \mathrm{COC}, \mathrm{SLL}, \mathrm{SD}, \mathrm{HHL}$ are or were employees, consultants and/or shareholders of Neuromod Devices. BL, SV, DAH serve on the clinical advisory board of Neuromod Devices and receive monetary compensation for their contribution. Data and materials availability: The study data pertaining to the results presented in this paper and supplementary materials can be accessed with reasonable request by contacting the corresponding author and with appropriate ethics committee and/or regulatory approvals. If there is requested data that is associated with proprietary information, these data can be made available after the corresponding patent filings within a reasonable timeframe. The clinical study is registered on clinicaltrials.gov (NCT02669069; (67)). 
Figure 1. Treatment approach and study design. (A) Schematic of CE marked bimodal neuromodulation device (marketed as Lenire) developed by Neuromod Devices (Dublin, Ireland) consisting of wireless (Bluetooth) headphones that deliver acoustic stimuli, a wire-connected 32electrode array for presenting electrical stimulation patterns to the anterior dorsal surface of the tongue, and a battery-powered controller that coordinates both stimulus modalities. Bimodal stimulation activates auditory and somatosensory pathways, as well as converging centers in auditory, limbic and attentional brain regions implicated in tinnitus. (B) Schematic of clinical study design in which 698 participants were screened at the clinic to enroll 326 eligible participants into one of three treatment arms. Participants were evaluated for changes in tinnitus symptom severity during the 12-week treatment period and at three follow-up visits up to 12-months post-treatment.
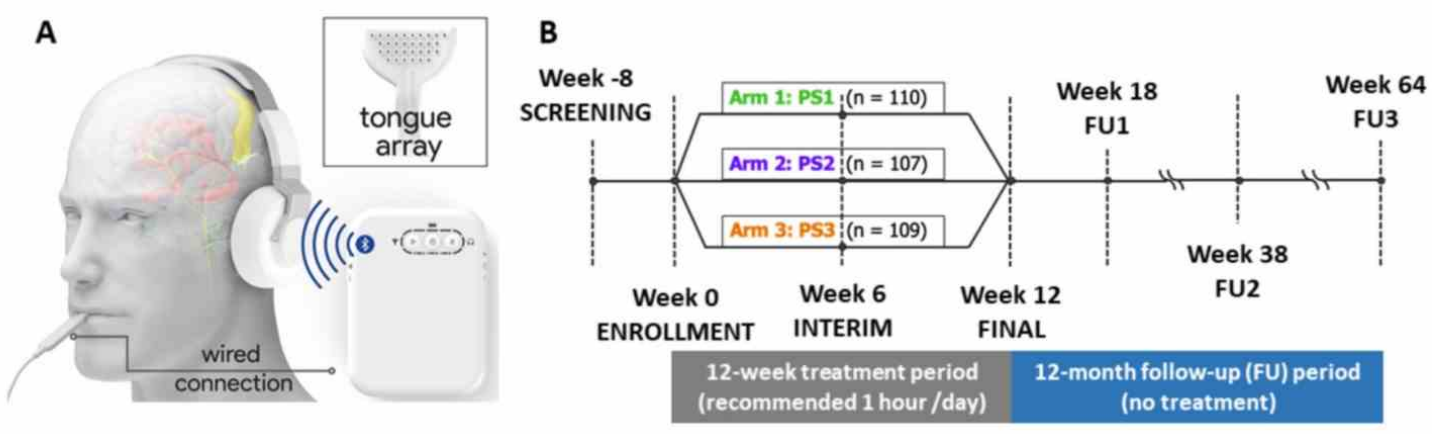
Note: Figure 2 is revised in the final published version.

Figure 2. Participant flow diagram. For the primary endpoints, within-arm comparisons were performed with a per-protocol analysis (PPA) for those who were compliant to treatment (device usage of at least 36 hours over the 12-week treatment period), while between-arm comparisons were performed with an intention-to-treat (ITT) analysis with imputation for missing values described in Materials and Methods. Follow-up (FU) visits are depicted in Fig. 1.

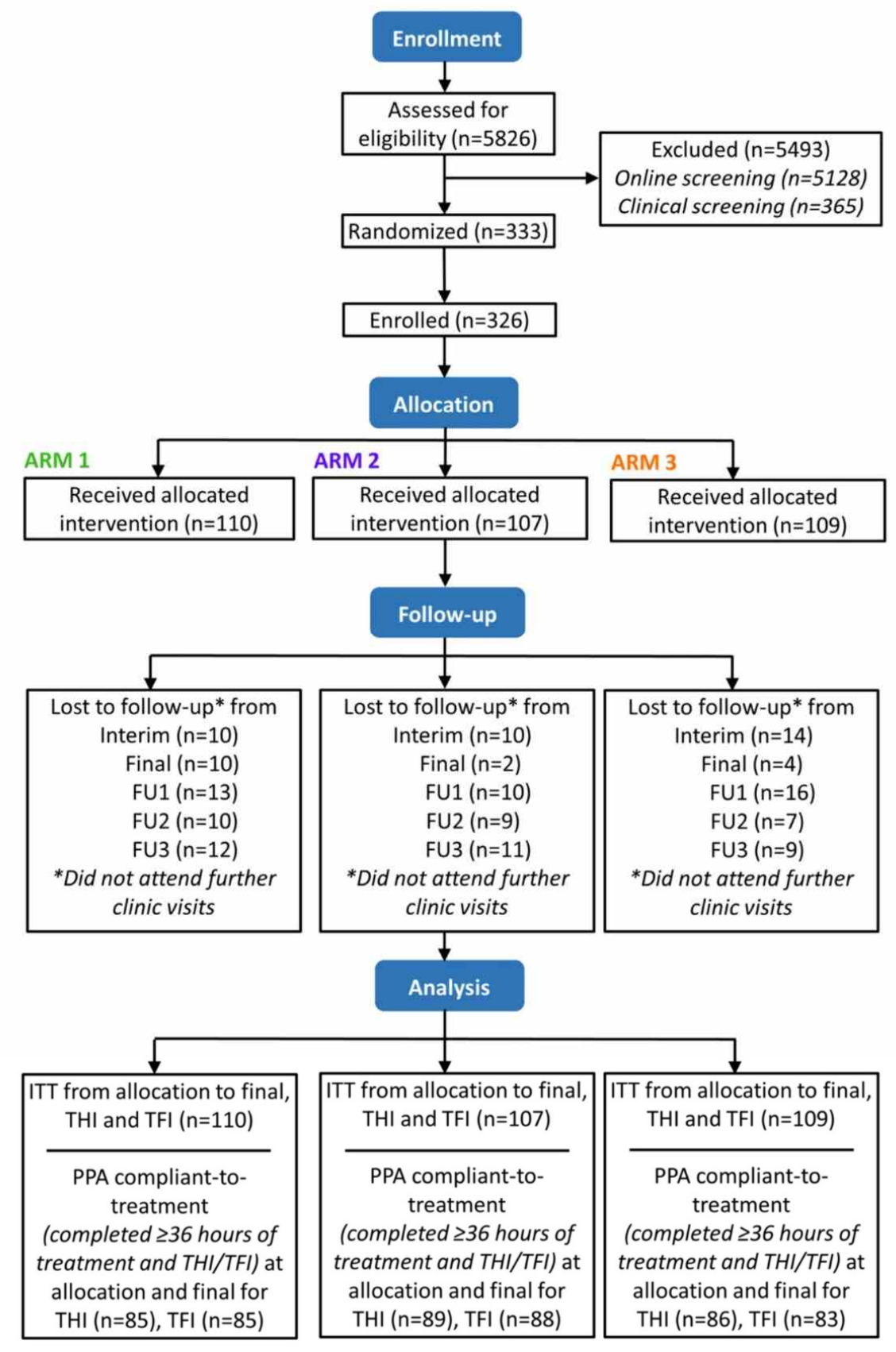


Figure 3. Changes in tinnitus symptom severity for each participant. (A) Scatter plots represent THI scores for each treatment-compliant individual at baseline (average of score at screening and enrollment) versus end of treatment (12-week "final" visit) for each arm. (B) Scatter plots represent TFI scores. Points below diagonal black line indicate an improvement (i.e., a decrease in score) in outcome measure of THI or TFI. Data points were jittered for visibility.

\section{A}
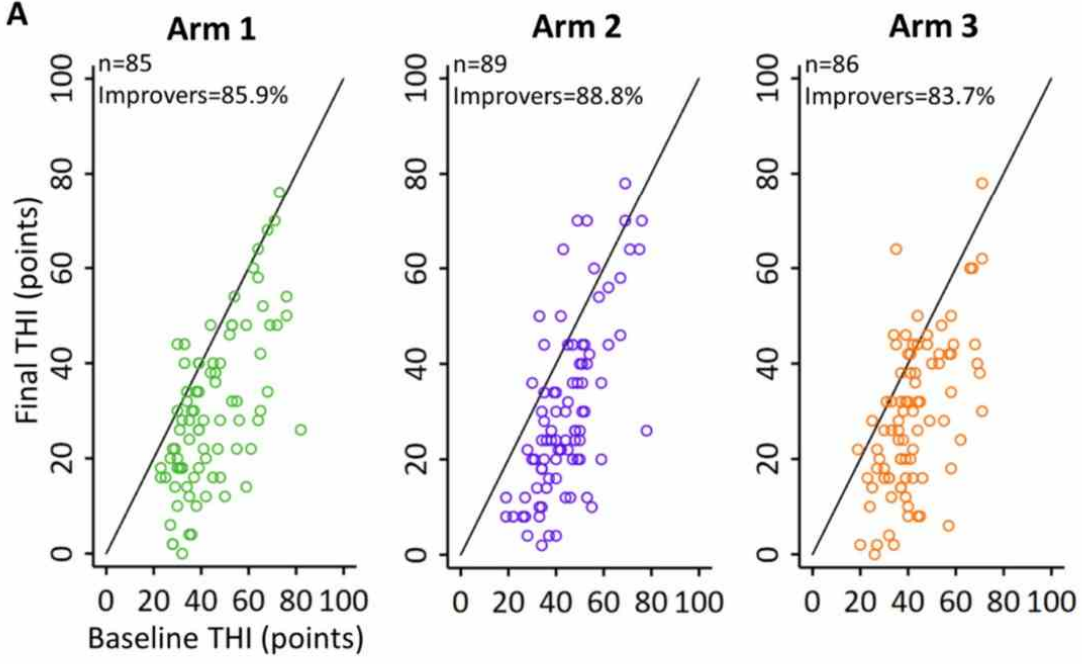

B

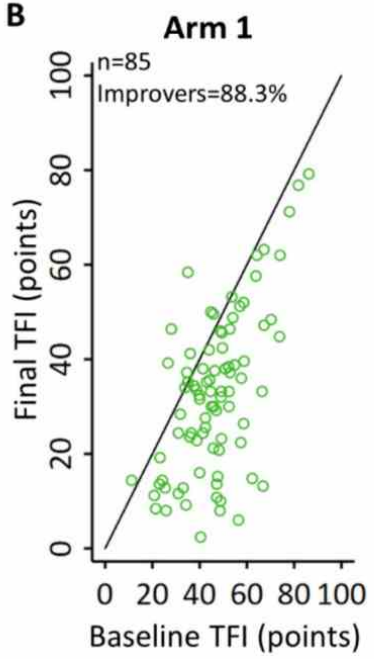

Arm 2
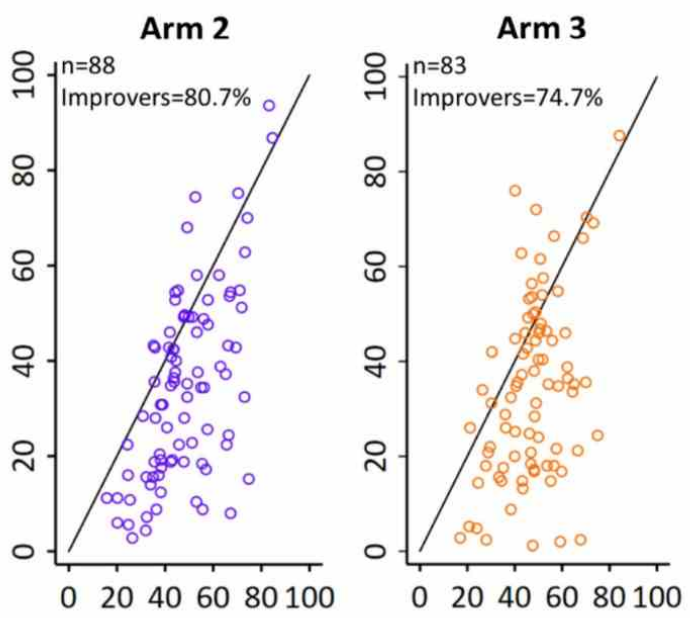
Figure 4. Within-arm and between-arm analyses for primary endpoints at 12-week assessment. The mean difference in THI or TFI score across participants for each arm from baseline to final assessment (end of treatment) for the within-arm cases, or the mean difference between two arms for the change in THI or TFI score from baseline to final assessment for the between-arm cases are plotted with the $\pm 95 \%$ confidence interval (CI). For the primary endpoints, the within-arm statistical analyses were based on a per-protocol estimand, which consists of all treatment-compliant participants with baseline and final scores. All within-arm comparisons for THI and TFI were highly significant based on paired two-tailed t-tests $(P<0.001 ; 95 \% \mathrm{CI}$ are nonoverlapping with the vertical line at zero). The between-arm statistical analyses were based on an intention-to-treat estimand and tested with multiple regression using baseline scores as a covariate. Missing data were handled by using a Markov chain Monte Carlo multiple imputation method (further details are provided in Materials and Methods), which leads to $n$ values that match the enrolled numbers for each arm. There were no significant differences between any arms $(P>0.05$; $95 \%$ CI crosses zero line).

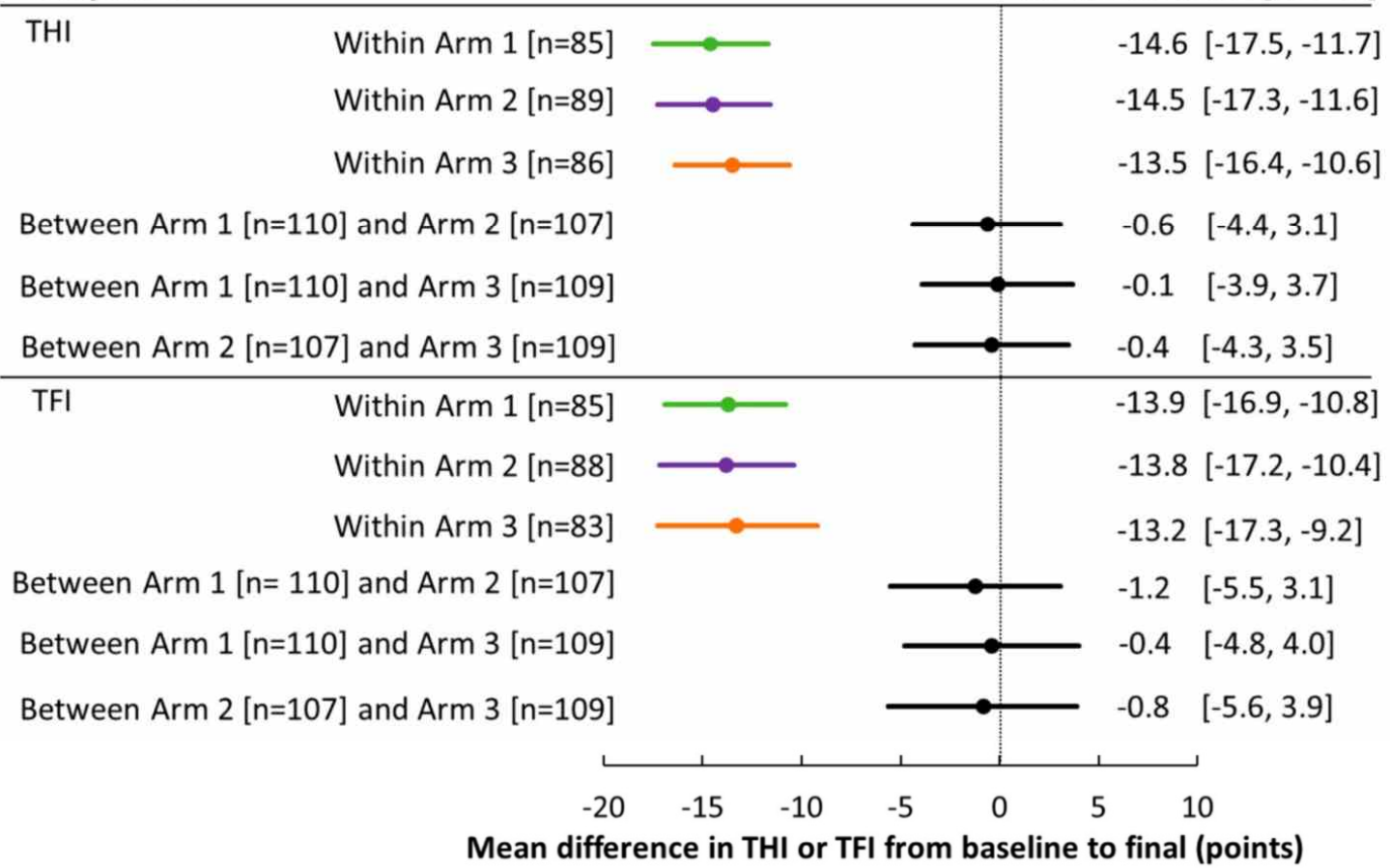


Figure 5. Long-term clinical efficacy across treatment arms. (A) Change in THI relative to baseline for all treatment-compliant participants who attended every assessment up to the 12month post-treatment visit (Week 64). The change in THI equals the global score (0 to 100) at a given time point minus the global score at baseline. Mean change values and $95 \%$ confidence intervals are plotted for each arm (Arm 1: $n=31$, Arm 2: $n=41$, Arm 3: $n=32$; same individuals at every visit for each arm). (B) Change in TFI relative to baseline for all treatment-compliant participants who attended every assessment up to the 12-month post-treatment visit. The change in TFI equals the global score ( 0 to 100$)$ at a given time point minus the global score at baseline. Mean change values and 95\% confidence intervals are plotted for each arm (Arm 1: $n=31$, Arm 2: $n=40$, Arm 3: $n=31$; same individuals at every visit for each arm). TFI assessment was not included in the protocol for the 38-week visit. During and after treatment, all arms exhibit a significant reduction in THI and TFI that is sustained out to 12-months post-treatment, except for Arm 3 at 6-weeks post-treatment for TFI ( $P>0.05 ; 95 \%$ confidence interval crosses the zero line). Based on post-hoc analyses, Arm 1 was significantly different than Arm 3 at 38-weeks (6-months post-treatment; $P=0.031)$ and at 64 -weeks $(12$-months post-treatment; $P=0.043)$ for THI. Arm 2 was significantly different than Arm 3 at 18 -weeks (6-weeks post-treatment; $P=0.030$ ) for THI. Statistical significance was determined using an unequal variance two-tailed t-test appropriate for these comparisons based on the method presented in (77). For clearer visualization, the data points and error bars were jittered in time. 


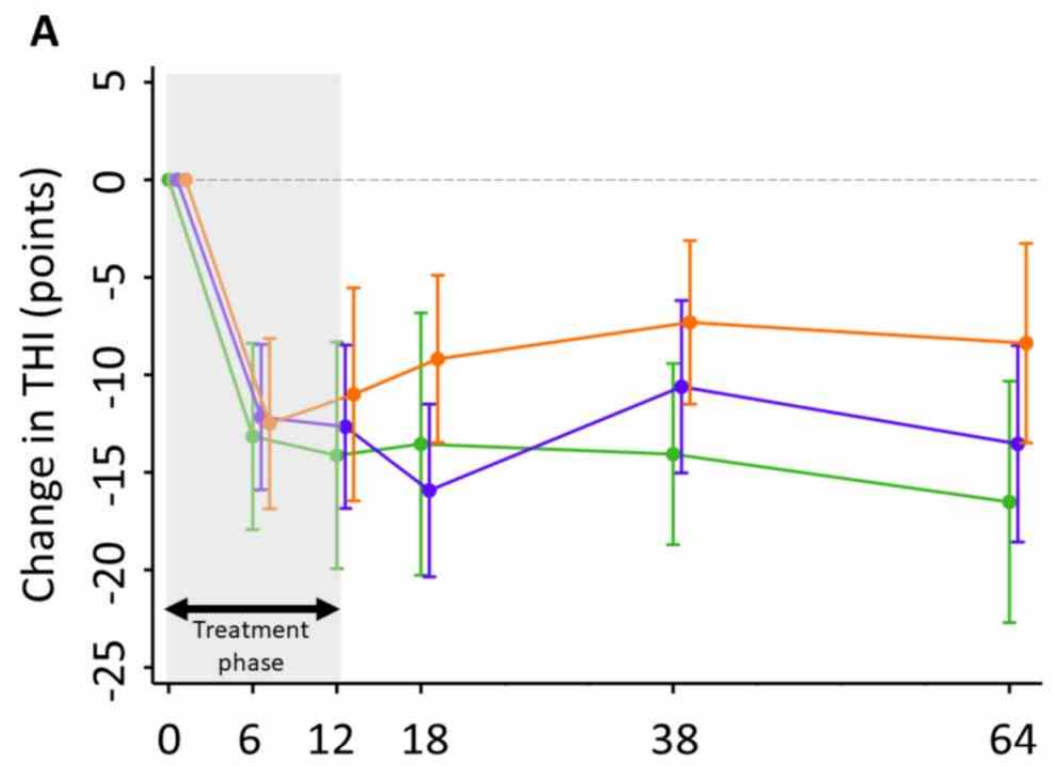

B

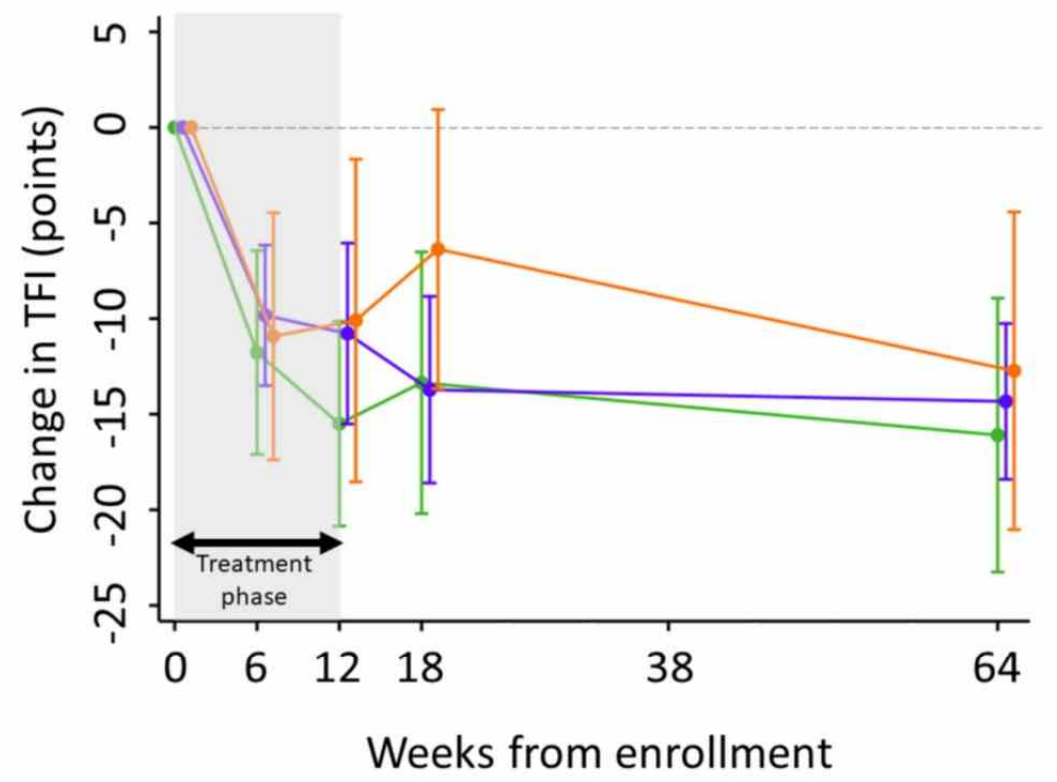

Arm $1 \square$ Arm $2 \square$ Arm 3 
Figure 6. Long-term clinical efficacy of bimodal neuromodulation. (A,B) Scatter plots represent THI or TFI scores for each treatment-compliant individual at baseline (average of score at screening and enrollment) versus end of treatment (12-week "final" visit) for all arms pooled together to represent bimodal neuromodulation in general. (C,D) Scatter plots represent THI or TFI scores for each treatment-compliant individual at baseline versus 12-month post-treatment visit for all arms pooled together. All individuals who completed at least the assessments displayed in each figure were included to maximize the total number of subjects plotted in each figure, leading to a large sample size for each plot. Data points are jittered for visibility.
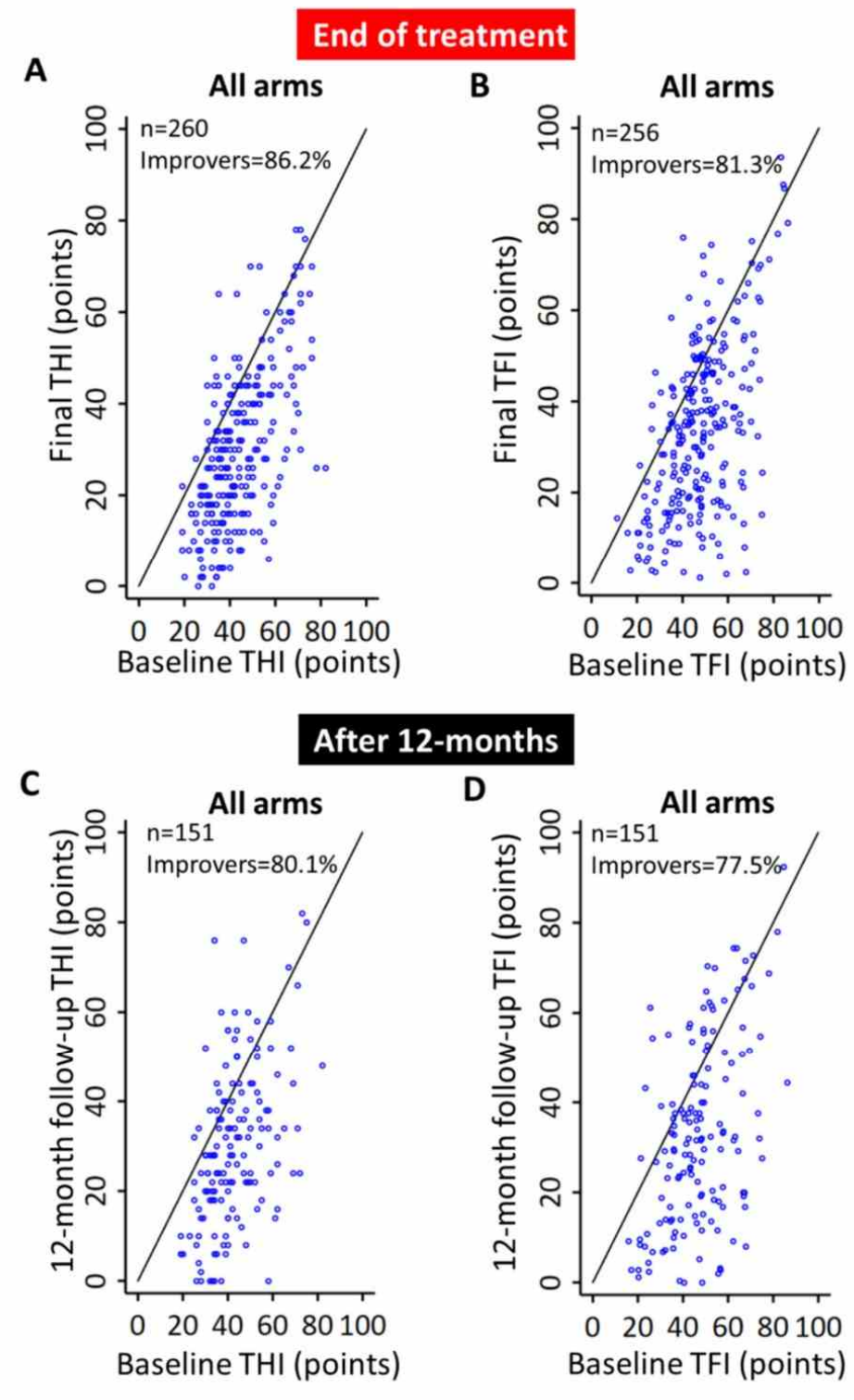
Table 1. Characteristics of enrolled participants and key numbers for each visit.

dB HL: decibel hearing level, dB SL: decibel sensation level (equals dB HL minus hearing threshold level of acoustic stimulus), FU: follow-up, LDL: loudness discomfort level, MML: minimum masking level. Somatic tinnitus is defined as a tinnitus that can be directly modulated by one or more head or neck maneuvers. MML was determined by presenting a wide-band noise at increasing intensities until the sound masked the participant's tinnitus. A description of the stratification categories is provided in Materials and Methods. P-value is based on the Fisher's exact test for count variables or a linear regression for continuous variables.

\begin{tabular}{|c|c|c|c|c|c|c|}
\hline Characteristics & Units & Full cohort & Arm 1 & Arm 2 & Arm 3 & P-value \\
\hline $\begin{array}{l}\text { total participants } \\
\text { enrolled (device fitted) }\end{array}$ & \# participants & 326 & 110 & 107 & 109 & 0.990 \\
\hline $\begin{array}{l}\text { Ireland: participants } \\
\text { enrolled (device fitted) }\end{array}$ & \# participants & 261 & 86 & 85 & 90 & 0.975 \\
\hline $\begin{array}{l}\text { Germany: participants } \\
\text { enrolled (device fitted) }\end{array}$ & \# participants & 65 & 24 & 22 & 19 & 0.883 \\
\hline gender: male & $\begin{array}{l}\text { \# participants } \\
\text { (\% of enrolled) }\end{array}$ & $212(65.0 \%)$ & 65 (59.1\%) & $79(73.8 \%)$ & $68(62.4 \%)$ & 0.694 \\
\hline gender: female & $\begin{array}{l}\text { \# participants } \\
\text { (\% of enrolled) }\end{array}$ & $114(35.0 \%)$ & 45 (40.9\%) & $28(26.2 \%)$ & $41(37.6 \%)$ & 0.351 \\
\hline age @ baseline & $\begin{array}{c}\text { years } \\
\text { [mean (SD)] }\end{array}$ & $48.1(11.6)$ & $46.3(12.0)$ & $48.7(12.6)$ & $49.5(10.0)$ & 0.102 \\
\hline tinnitus duration & $\begin{array}{c}\text { years } \\
\text { [mean (SD)] }\end{array}$ & $2.6(1.6)$ & $2.5(1.7)$ & $2.7(1.6)$ & $2.6(1.6)$ & 0.777 \\
\hline THI@ baseline & $\begin{array}{c}\text { points } \\
\text { [mean (SD)] }\end{array}$ & $43.5(13.2)$ & $44.5(14.2)$ & $44.0(12.7)$ & $42.0(12.6)$ & 0.325 \\
\hline TFI @ baseline & $\begin{array}{c}\text { points } \\
{[\text { mean }(S D)]}\end{array}$ & $47.9(14.7)$ & $47.7(14.5)$ & $48.1(14.9)$ & $47.7(14.9)$ & 0.969 \\
\hline $\begin{array}{c}\text { mean hearing loss } \\
(250 \mathrm{~Hz} \text { to } 8 \mathrm{kHz}) @ \text { screening }\end{array}$ & $\begin{array}{c}\mathrm{dB} \mathrm{HL} \\
{[\text { mean (SD)] }}\end{array}$ & $17.8(10.3)$ & $17.2(9.9)$ & $18.1(9.7)$ & $18.2(11.2)$ & 0.699 \\
\hline attended interim visit & $\begin{array}{l}\text { \# participants } \\
\text { (\% of enrolled) }\end{array}$ & $277(85.0 \%)$ & 97 (88.2\%) & 92 (86.0\%) & $88(80.7 \%)$ & 0.898 \\
\hline attended final visit & $\begin{array}{l}\text { \# participants } \\
\text { (\% of enrolled) }\end{array}$ & $274(84.0 \%)$ & $89(80.9 \%)$ & $94(87.9 \%)$ & $91(83.5 \%)$ & 0.964 \\
\hline attended FU1 visit & $\begin{array}{l}\text { \# participants } \\
\text { (\% of enrolled) }\end{array}$ & $185(56.8 \%)$ & $59(53.6 \%)$ & $70(66.4 \%)$ & $56(51.4 \%)$ & 0.620 \\
\hline attended FU2 visit & $\begin{array}{l}\text { \# participants } \\
\text { (\% of enrolled) }\end{array}$ & $183(56.1 \%)$ & $57(51.8 \%)$ & $69(64.5 \%)$ & $57(52.3 \%)$ & 0.716 \\
\hline attended FU3 visit & $\begin{array}{l}\text { \# participants } \\
\text { (\% of enrolled) }\end{array}$ & $156(47.9 \%)$ & $46(41.8 \%)$ & $57(53.3 \%)$ & $53(48.6 \%)$ & 0.760 \\
\hline \multicolumn{7}{|l|}{$\underline{\text { Stratification }}$} \\
\hline atonal tinnitus & $\begin{array}{l}\text { \# participants } \\
\text { (\% of enrolled) }\end{array}$ & 50 (15.3\%) & $18(16.4 \%)$ & $16(15.0 \%)$ & $16(14.7 \%)$ & 1.000 \\
\hline $\begin{array}{c}\text { hyperacusis } \\
\text { (LDL <60dB SL @ 500Hz) }\end{array}$ & $\begin{array}{l}\text { \# participants } \\
\text { (\% of enrolled) }\end{array}$ & $66(20.2 \%)$ & 19 (17.3\%) & $21(19.6 \%)$ & $26(23.9 \%)$ & 0.743 \\
\hline $\begin{array}{c}\text { normal hearing } \\
(<=20 \mathrm{~dB} \mathrm{HL}, 250 \mathrm{~Hz} \text { to } 8 \mathrm{kHz})\end{array}$ & $\begin{array}{l}\text { \# participants } \\
\text { (\% of enrolled) }\end{array}$ & 78 (23.9\%) & $26(23.6 \%)$ & $26(24.3 \%)$ & $26(23.9 \%)$ & 1.000 \\
\hline
\end{tabular}




\begin{tabular}{|c|c|c|c|c|c|c|}
\hline noise induced hearing loss & $\begin{array}{c}\text { \# participants } \\
\text { (\% of enrolled) }\end{array}$ & $107(32.8 \%)$ & $35(31.8 \%)$ & $37(34.6 \%)$ & $35(32.1 \%)$ & 1.000 \\
\hline Other groups & & & & & & \\
\hline $\begin{array}{c}\text { somatic tinnitus } \\
(>=1 \text { maneuver) }\end{array}$ & $\begin{array}{c}\text { \# participants } \\
\text { (\% of enrolled) }\end{array}$ & $201(61.7 \%)$ & $67(60.9 \%)$ & $63(58.9 \%)$ & $71(65.1 \%)$ & 0.906 \\
\hline $\begin{array}{c}\text { substantial MML } \\
(>=15 \mathrm{~dB} S \mathrm{SL})\end{array}$ & $\begin{array}{c}\text { \# participants } \\
\text { (\% of enrolled) }\end{array}$ & $240(73.6 \%)$ & $80(72.7 \%)$ & $84(78.5 \%)$ & $76(69.7 \%)$ & 0.902 \\
\hline $\begin{array}{c}\text { tinnitus duration } \\
(<3 \text { years })\end{array}$ & $\begin{array}{c}\text { \# participants } \\
(\% \text { of enrolled) }\end{array}$ & $170(52.1 \%)$ & $58(52.7 \%)$ & $54(50.5 \%)$ & $58(53.2 \%)$ & 0.953 \\
\hline
\end{tabular}


Table 2. Safety data recorded throughout study.

A

\begin{tabular}{|c|c|c|c|}
\hline Potential treatment adverse events & Total & Negligible & Moderate \\
\hline (numbers based on 326 enrolled participants) & 155 & 96 & 59 \\
\hline Probably device related & 35 & 19 & 16 \\
\hline subjective increase in tinnitus symptoms & 9 & 3 & 6 \\
\hline dramatic increase in tinnitus symptoms & 2 & 0 & 2 \\
\hline fluctuating tinnitus & 1 & 0 & 1 \\
\hline headache or pain in the head area & 1 & 0 & 1 \\
\hline pain in the ear or mouth area & 4 & 4 & 0 \\
\hline temporary swelling of gums & 1 & 1 & 0 \\
\hline sensitivity of tongue or oral mucosa & 2 & 2 & 0 \\
\hline transient discomfort associated with tongue part & 2 & 2 & 0 \\
\hline ulceration of oral cavity & 2 & 1 & 1 \\
\hline ulceration of the tongue & 4 & 1 & 3 \\
\hline unanticipated adverse event [see B] & 2 & 1 & 1 \\
\hline mis-use of device * & 5 & 4 & 1 \\
\hline Possibly device related & 85 & 46 & 39 \\
\hline subjective increase in tinnitus symptoms & 45 & 26 & 19 \\
\hline dramatic increase in tinnitus symptoms & 3 & 0 & 3 \\
\hline fluctuating tinnitus & 9 & 5 & 4 \\
\hline headache or pain in the head area & 3 & 2 & 1 \\
\hline pain in the ear or mouth area & 6 & 4 & 2 \\
\hline transient discomfort associated with tongue part & 2 & 2 & 0 \\
\hline ulceration of oral cavity & 2 & 0 & 2 \\
\hline ulceration of the tongue & 1 & 0 & 1 \\
\hline unanticipated adverse event [see B] & 11 & 5 & 6 \\
\hline event cause by patient conditions [see text] & 3 & 2 & 1 \\
\hline Probably not device related & 35 & 31 & 4 \\
\hline subjective increase in tinnitus symptoms & 3 & 3 & 0 \\
\hline dramatic increase in tinnitus symptoms & 2 & 0 & 2 \\
\hline pain in the ear or mouth area & 1 & 1 & 0 \\
\hline increase in hearing threshold \# & 1 & 1 & 0 \\
\hline unanticipated adverse event [see B] & 4 & 4 & 0 \\
\hline event caused by patient conditions [see text] & 24 & 22 & 2 \\
\hline
\end{tabular}

B

\begin{tabular}{|l|l|l|}
\hline \multicolumn{3}{|c|}{ Unanticipated adverse events } \\
\hline Probably device related & Possibly device related & Probably not device related \\
Moderate: & Moderate: & Negligible: \\
\hline $\begin{array}{l}\text { - small red fissure on } \\
\text { right buccal mucosa }\end{array}$ & - cold sores (4 cases) & - ulceration of lip but not \\
Negligible: & - angular cheilitis & reported during \\
\hline - metallic taste from & - increased nervousness and & treatment \\
tongue component & shakiness & - pooling of saliva and \\
& Negligible: & difficulty swallowing but \\
& - light headedness for a few & participant does not \\
& seconds & believe due to device \\
& - temporary dizziness & - difficulty sleeping but \\
& - fullness in ears for a few & reported a cold during \\
& seconds & the same period \\
& - tingling in ears periodically & - difficulty sleeping but \\
& - temporary tiredness and & reported cessation of \\
& nausea & alcohol intake \\
\hline
\end{tabular}


* Moderate case caused by an individual overly increasing sound volume to attempt to mask the tinnitus that led to a bothersome increase in tinnitus, but individual was instructed to reduce loudness to a comfortable intensity that improved the situation. There were four negligible adverse events in which participants increased tongue stimulation intensity too high that caused discomfort in the oral cavity and tongue area, in which they were instructed to reduce the intensity to improve situation.

\# One individual had a $40 \mathrm{~dB}$ increase in hearing threshold at $1 \mathrm{kHz}$ in the right ear between Screening and Final assessments, but re-measuring thresholds at 6-week follow-up showed same thresholds as Screening visit. Since there were no obvious cases of compromised hearing across participants attributed to the treatment, this case was likely attributed to a data entry error. 


\section{$\underline{\text { Supplementary Materials }}$}

A Compliance rates

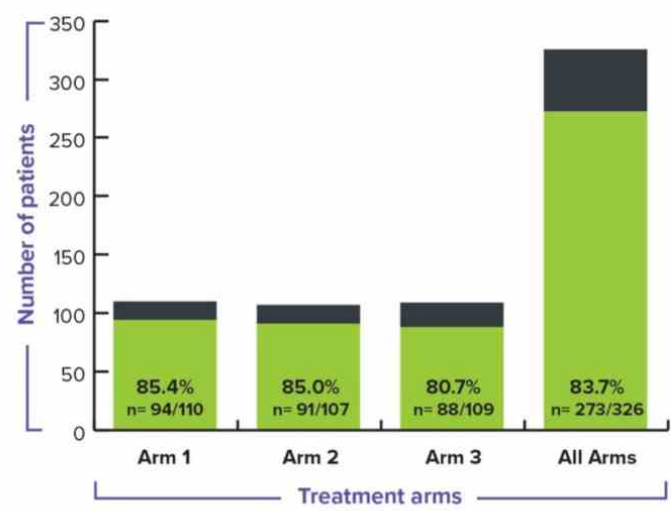

B

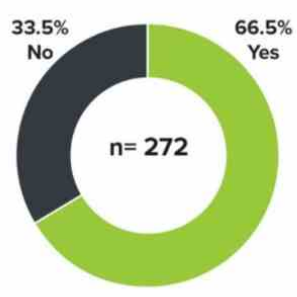

Overall, would you say you have benefitted
from using the device?

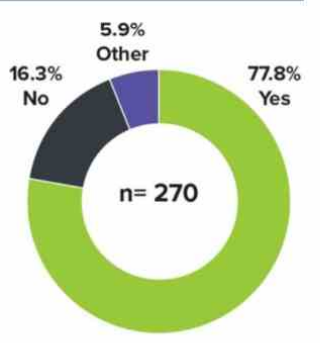

If you knew someone with tinnitus would you recommend

Fig. S1. Compliance and satisfaction rates in using treatment device. (A) Number of enrolled participants who achieved the minimum treatment compliance of $\geq 36$ hours over the intended 12 week treatment period (41). The $n$ values are larger in this figure compared to the $n$ values for the per-protocol analysis in Fig. 2 because Fig. 2 includes treatment-compliant participants who also came to the 12-week visit for THI and TFI assessments. Since the device tracks when it is used by the participants, we were able to determine if participants were compliant to treatment once they returned their device, in which some participants did not attend the 12-week visit but returned the device afterwards. There was no statistically significant differences in compliance rate or number of participants at the final visit between treatment arms $(P>0.05$; Fisher's exact test). (B) Two questions relating to the participant's satisfaction or acceptability of the treatment device were asked at the 12-week visit, in which the percentage of YES or NO responses are shown. The "other" category refers to cases where the participant could not answer YES or NO or did not feel comfortable in committing to an answer. 


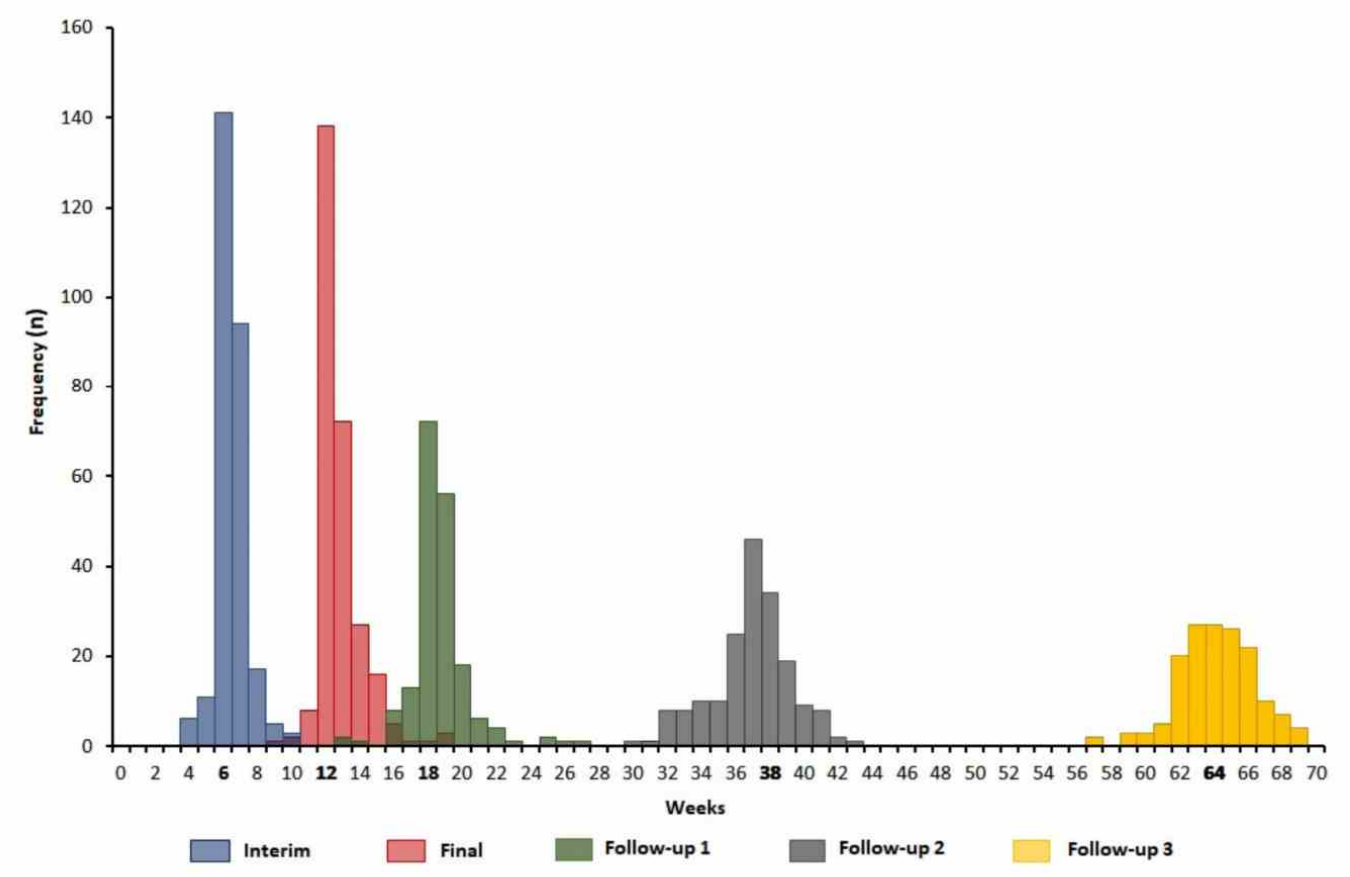

Fig. S2. Histogram of time (in weeks) when participants attended each visit relative to enrollment. Treatment began at $t=0$ weeks. The participants generally attended each visit at or near the intended time point of 6, 12, 18, 38 and 64 weeks relative to enrollment (see Fig. 1 for study timeline). 

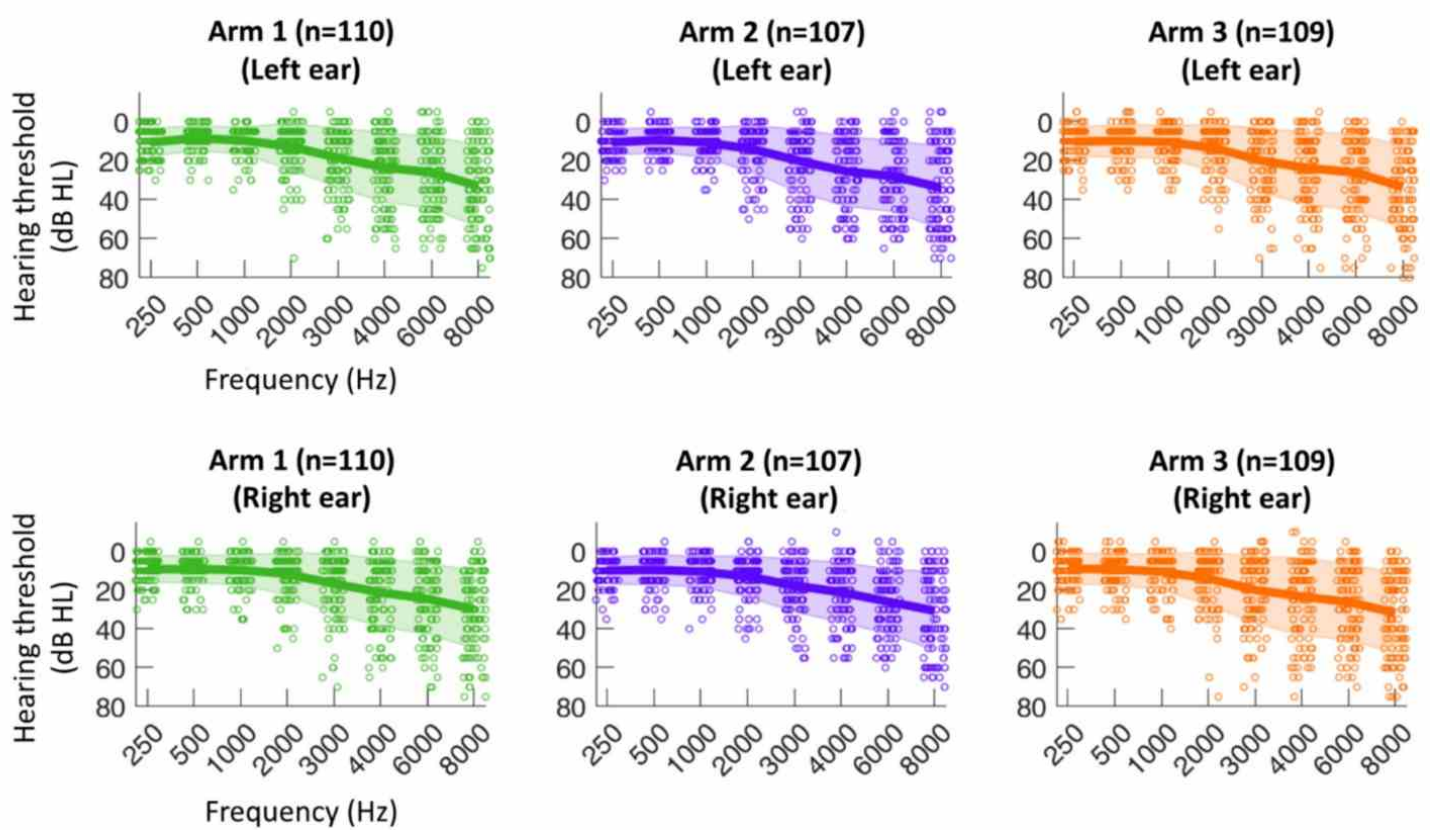

Fig. S3. Hearing thresholds for treatment-compliant participants. Data are plotted for each arm or grouped together and presented for left or right ear. Circle represents threshold value for each frequency and participant, solid line corresponds to mean threshold value for each frequency across participants, and shaded region corresponds to standard deviation of threshold values for each frequency across participants. Data points are jittered for visibility. dB HL: decibels in hearing level. 
A

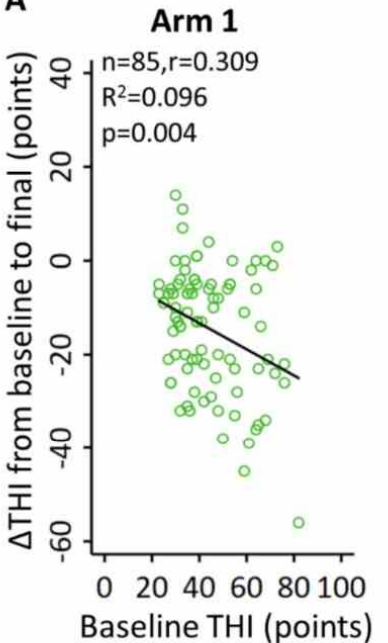

B

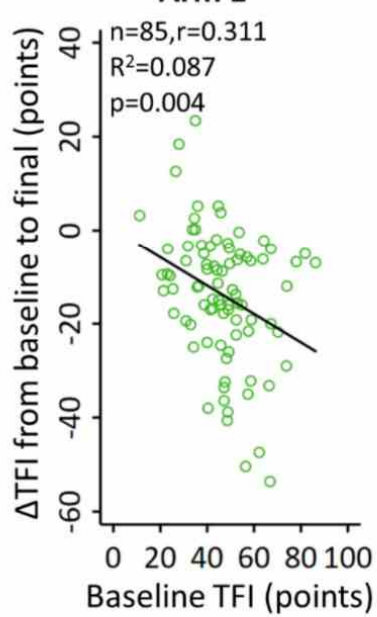

Arm 2

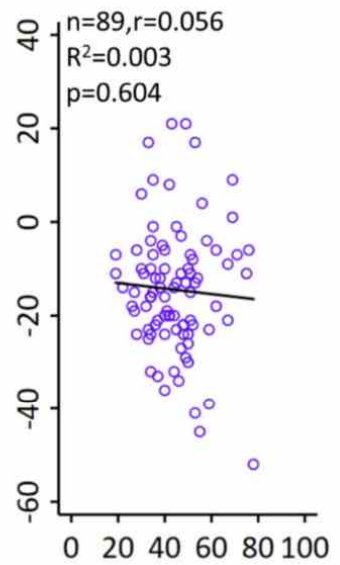

Arm 2

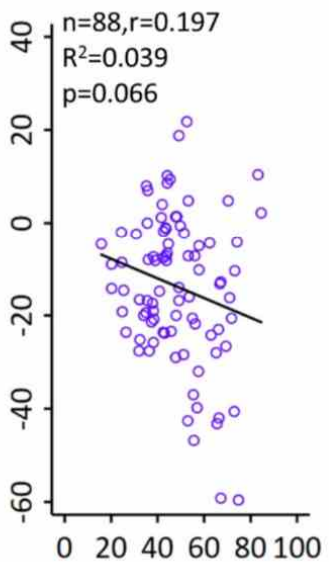

Arm 3

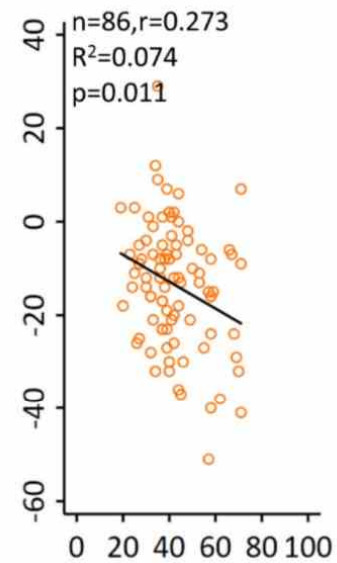

Arm 3

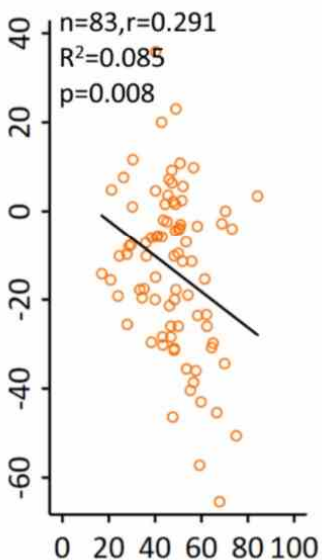

Fig. S4. Scatter plots for change in THI or TFI score. Change measured from baseline to end of 12-week treatment versus baseline (A) THI or (B) TFI score for each arm. These data correspond to the participants included in Fig. 3. A linear regression was performed for each plot. Data points are jittered for visibility. $\mathrm{r}$ is listed as absolute value. 

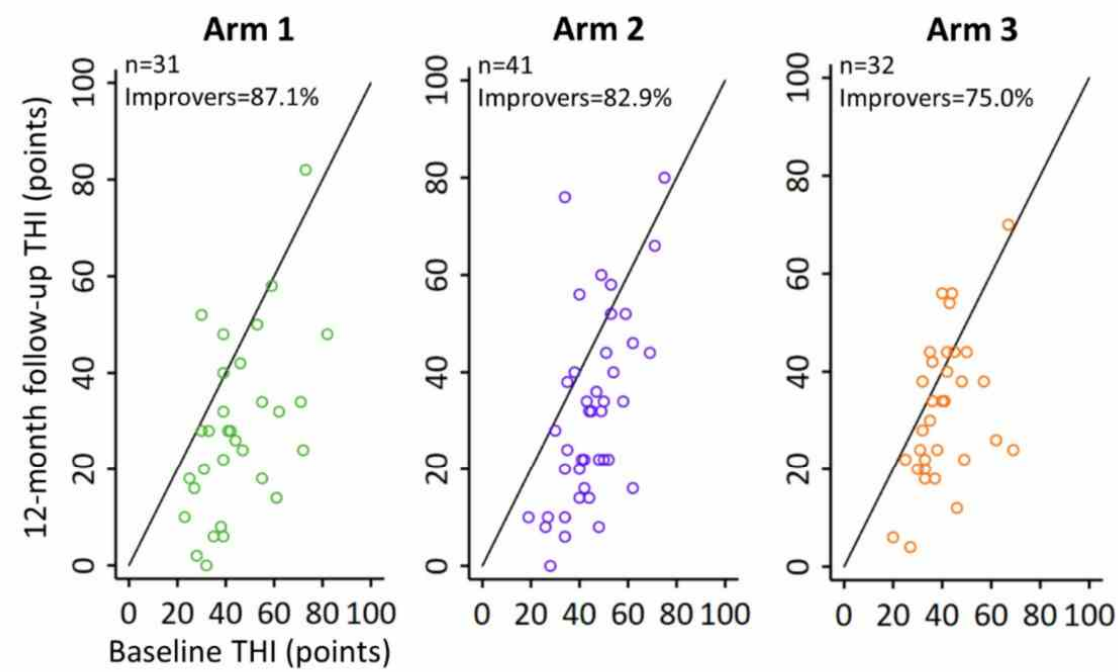

Fig. S5. Scatter plots for THI scores at baseline versus 12-month post-treatment assessment for each arm. Figure corresponds to participants included in Fig. 5. Circles below the black diagonal line correspond to "Improvers", which is defined as participants who retained a reduction in THI score relative to baseline. The proportion of participants retaining an improvement in tinnitus symptoms is greater in Arm 1 (87\%) and Arm 2 (83\%) compared to Arm 3 (75\%). Based on the Fisher's Exact Test, these percentages were not significantly different across arms $(P>$ $0.05)$. Data points are jittered for visibility. 

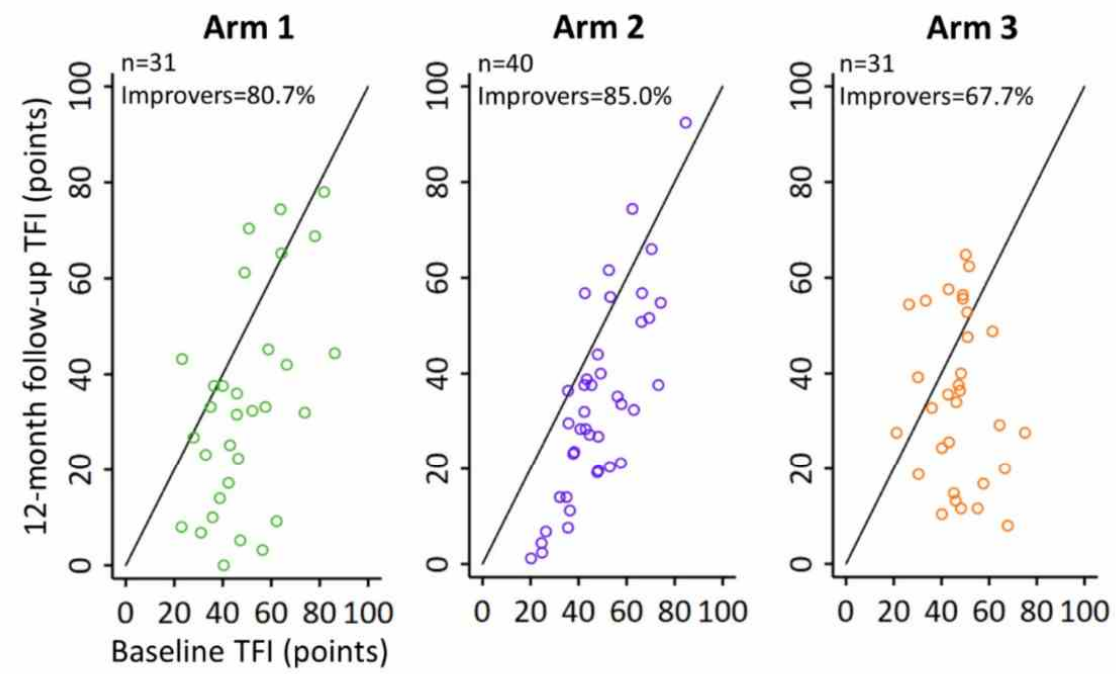

Fig. S6. Scatter plots for TFI scores at baseline versus 12-month post-treatment assessment for each arm. Figure corresponds to participants included in Fig. 5. Circles below the black diagonal line correspond to "Improvers", which is defined as participants who retained a reduction in TFI score relative to baseline. The proportion of participants retaining an improvement in tinnitus symptoms is greater in Arm 1 (81\%) and Arm 2 (85\%) compared to Arm $3(68 \%)$. Based on the Fisher's Exact Test, these percentages were not significantly different across arms $(P>$ $0.05)$. Data points are jittered for visibility. 
A
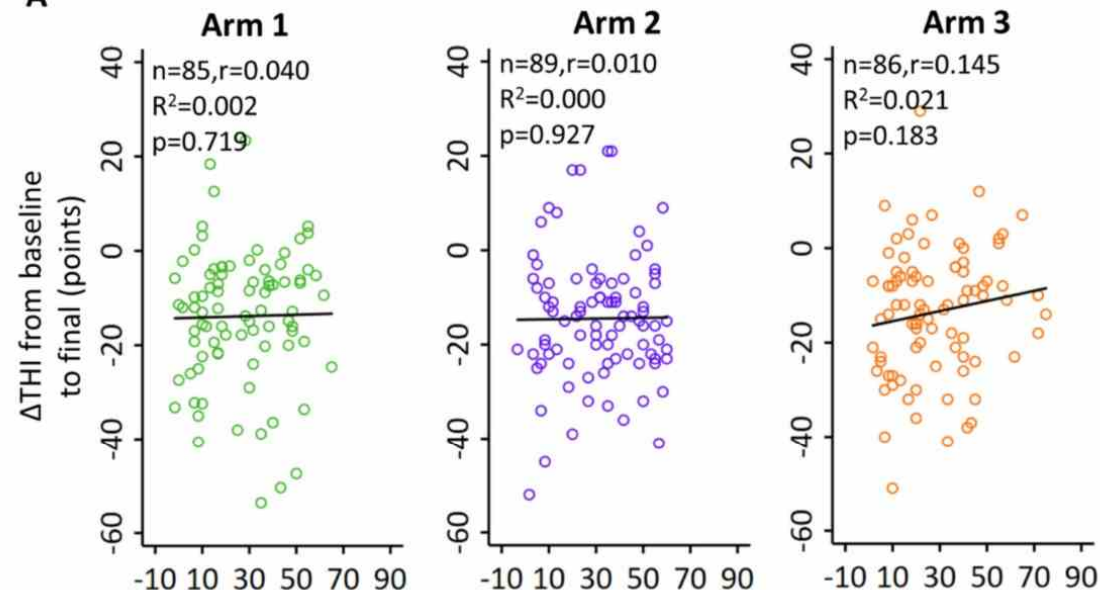

High frequency hearing loss

(mean $\mathrm{dB} \mathrm{HL}$ across 4 to $8 \mathrm{kHz}$ )

B
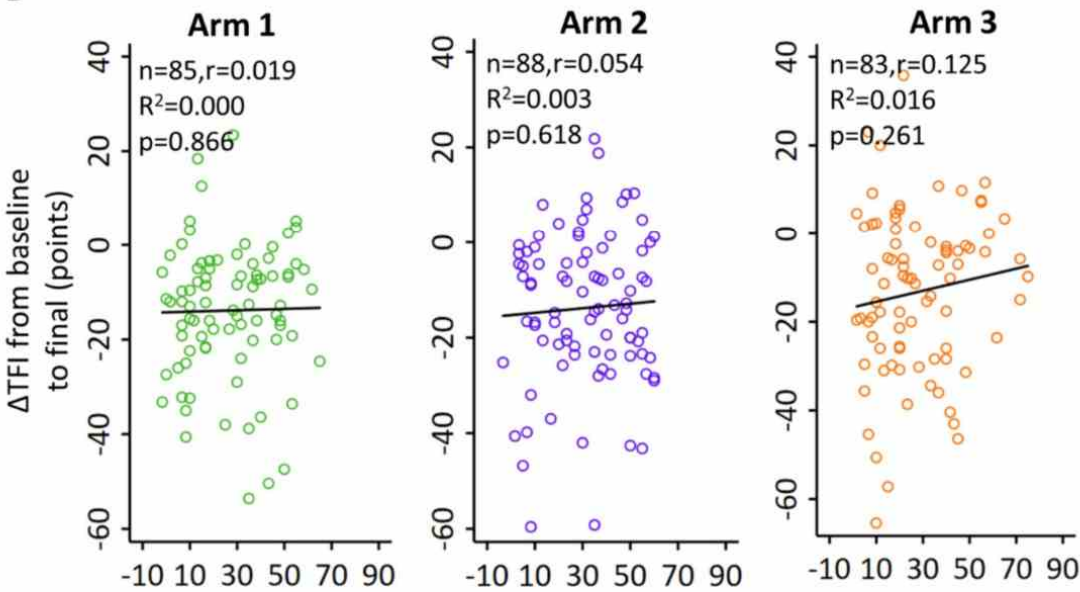

High frequency hearing loss

(mean dB HL across 4 to $8 \mathrm{kHz}$ )

Fig. S7. Scatter plots for change in THI or TFI score (from baseline to end of 12-week treatment) versus high frequency hearing loss $(4 \mathrm{to} 8 \mathrm{kHz})$ for each $\mathrm{arm}$. These data correspond to the participants included in Fig. 3 related to (A) THI and (B) TFI. A linear regression was performed for each plot. The high frequency hearing loss value is the mean value (dB HL) across several frequencies (4, 6 and $8 \mathrm{kHz}$; worst ear per frequency) corresponding to the portion of the audiograms in fig. S3 with greater hearing loss values. Data points are jittered for visibility. $r$ is listed as absolute value. 
A

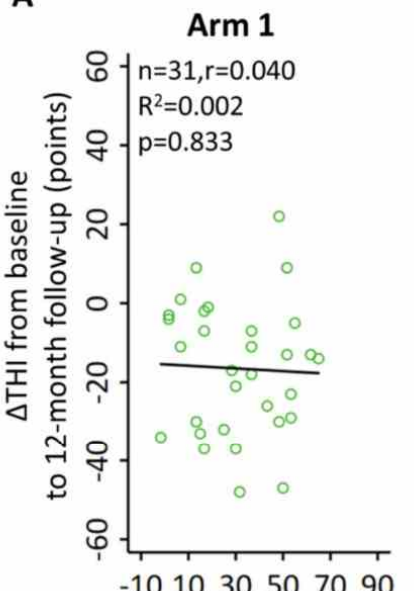

Arm 2

Arm 3

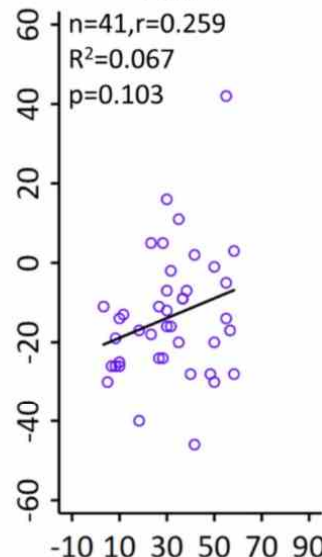

High frequency hearing loss

(mean dB HL across 4 to $8 \mathrm{kHz}$ )

B

Arm 1

Arm 2
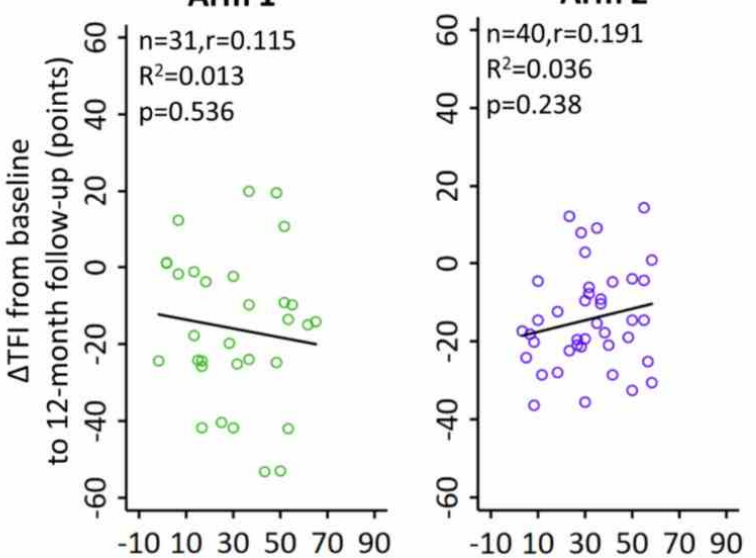

High frequency hearing loss

(mean $\mathrm{dB} \mathrm{HL}$ across 4 to $8 \mathrm{kHz}$ )
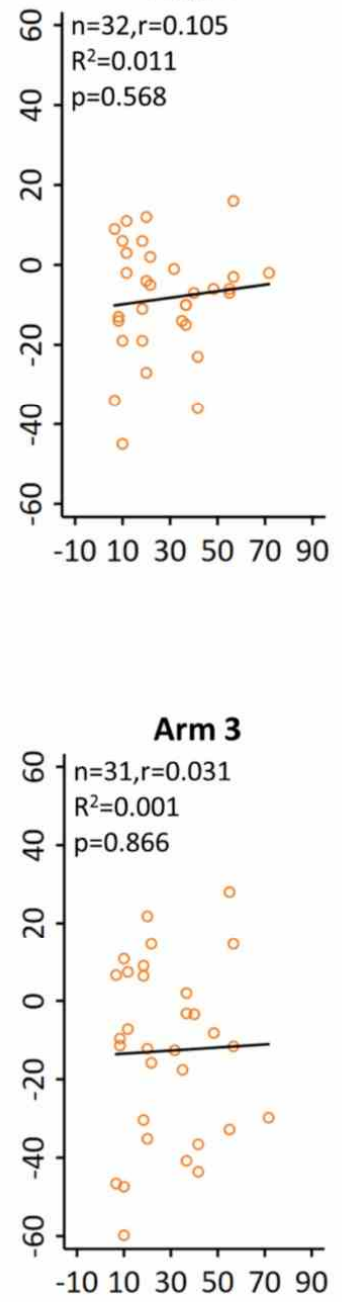

Fig. S8. Scatter plots for change in THI or TFI score (from baseline to 12-month posttreatment assessment) versus high frequency hearing loss $(4 \mathrm{to} 8 \mathrm{kHz})$ for each $\mathrm{arm}$. These data correspond to the participants included in Fig. 5 for (A) THI and (B) TFI. A linear regression was performed for each plot. The high frequency hearing loss value is the mean value (dB HL) across several frequencies (4, 6 and $8 \mathrm{kHz}$; worst ear per frequency) corresponding to the portion of the audiograms in fig. S3 with greater hearing loss values. Data points are jittered for visibility. $r$ is listed as absolute value. 
A

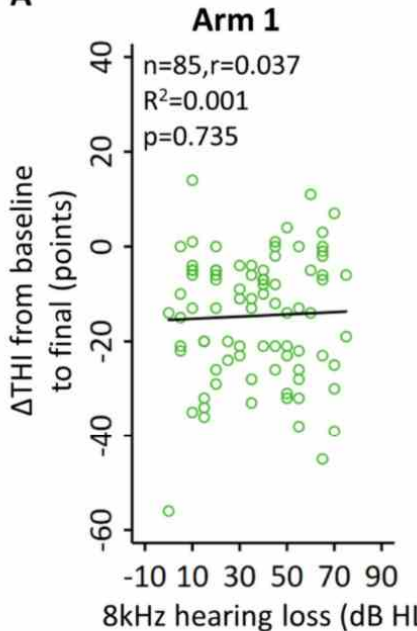

Arm 2

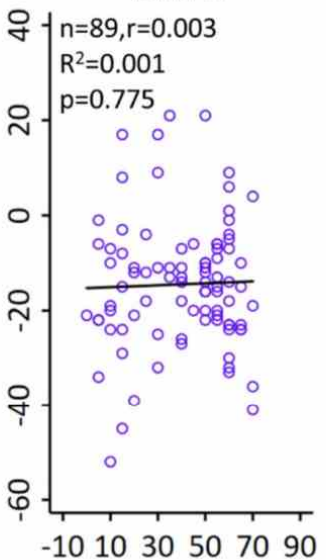

Arm 3

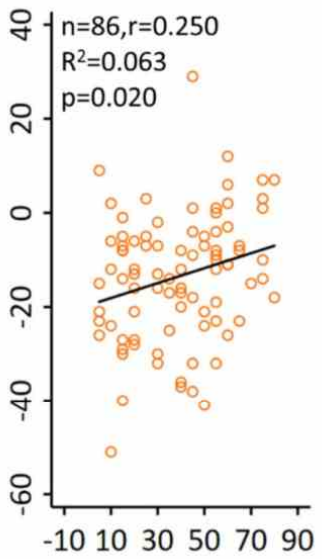

B

Arm 1

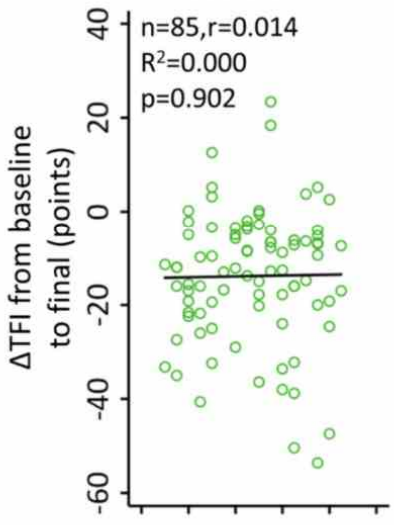

$-101030507090$ $8 \mathrm{kHz}$ hearing loss ( $\mathrm{dB} \mathrm{HL})$
Arm 2

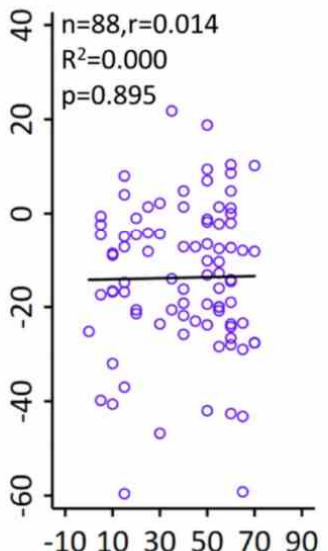

$-101030507090$

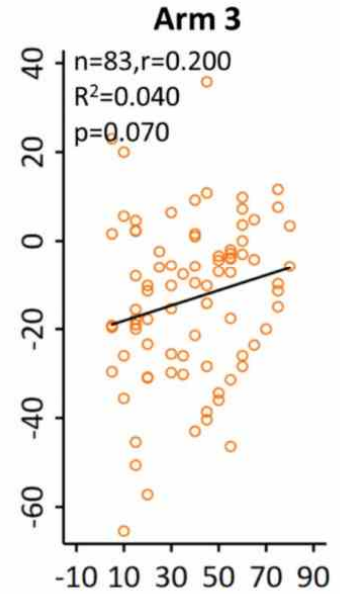

Fig. S9. Scatter plots for change in THI or TFI score (from baseline to end of 12-week treatment) versus $8 \mathrm{kHz}$ hearing loss for each arm. These data correspond to the participants included in Fig. 3 for (A) THI and (B) TFI. A linear regression was performed for each plot. Only $8 \mathrm{kHz}$ hearing loss (worst ear) is included in these plots to assess the trends for the highest frequency with the greatest hearing loss values assessed in this study (see fig. S3). Data points are jittered for visibility. $r$ is listed as absolute value. 
A

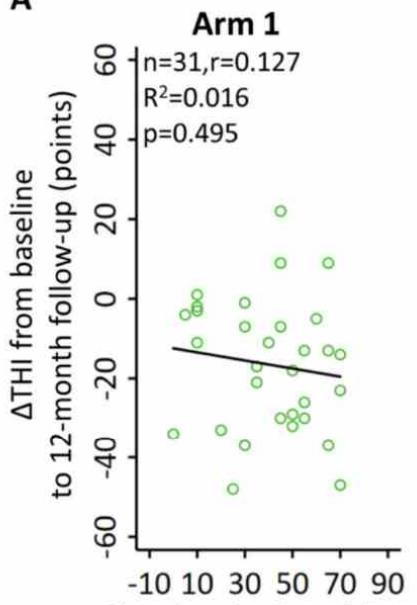

$8 \mathrm{kHz}$ hearing loss $(\mathrm{dB} \mathrm{HL})$
Arm 2

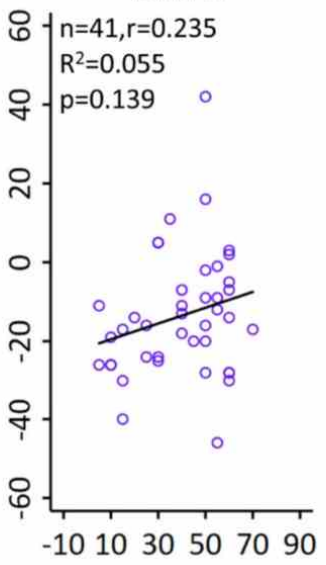

$-101030507090$

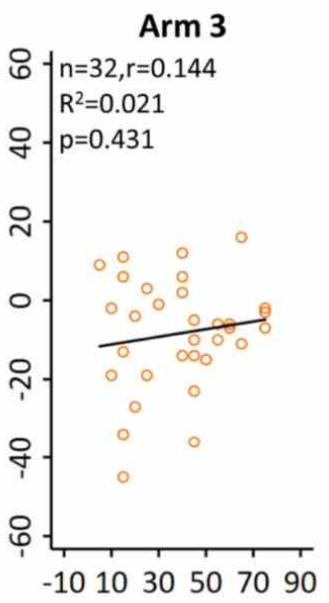

B

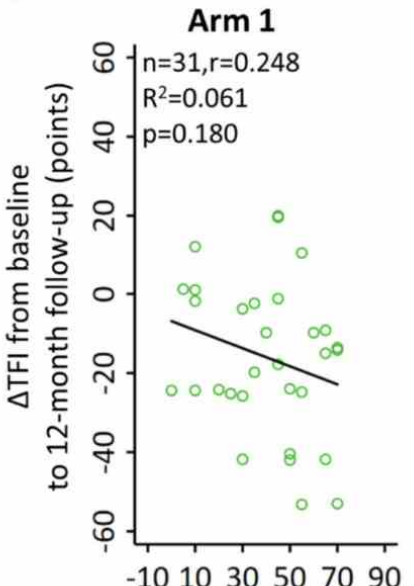

$8 \mathrm{kHz}$ hearing loss ( $\mathrm{dB} \mathrm{HL})$

Arm 2

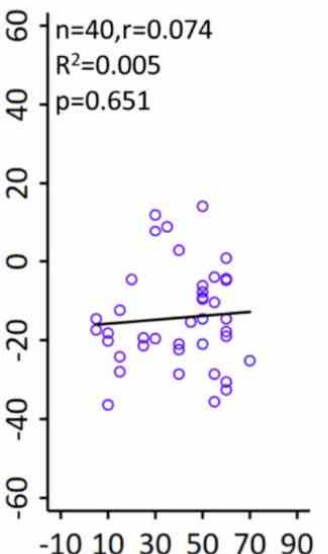

$-101030507090$

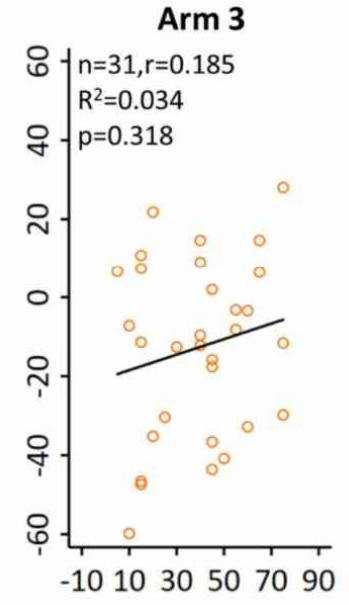

Fig. S10. Scatter plots for change in THI or TFI score (from baseline to 12-month posttreatment) versus $8 \mathrm{kHz}$ hearing loss for each arm. These data correspond to the participants included in Fig. 5 for (A) THI and (B) TFI. A linear regression was performed for each plot. Only $8 \mathrm{kHz}$ hearing loss (worst ear) is included in these plots to assess the trends for the highest frequency with the greatest hearing loss values assessed in this study (see fig. S3). Data points are jittered for visibility. $r$ is listed as absolute value. 

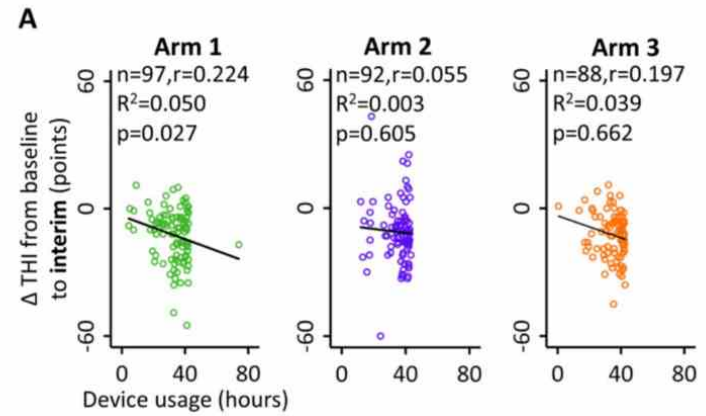

B
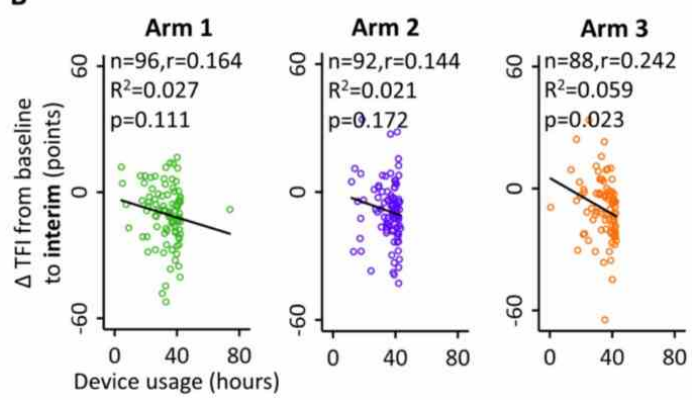

C
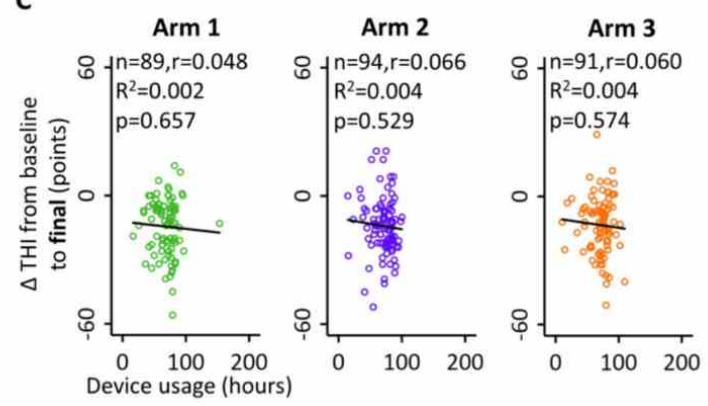

D
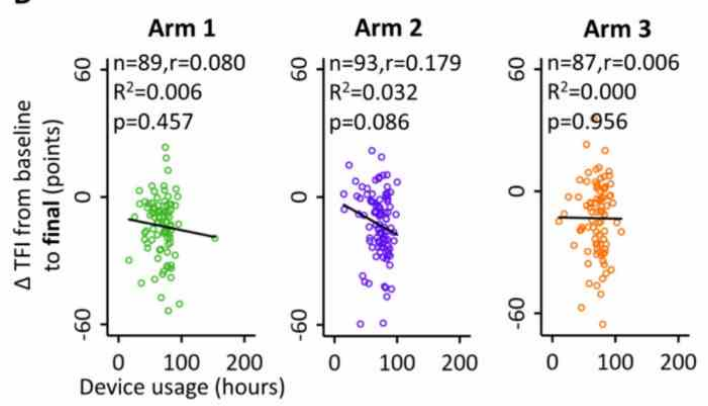

Fig. S11. Scatter plots for change in THI or TFI score versus duration of device usage. $(A, B)$ Change in THI or TFI score from baseline to interim assessment versus device usage during the first 6-weeks of treatment is plotted for each participant and arm. (C,D) Change in THI or TFI score from baseline to final assessment versus device usage during the full 12-week treatment period is plotted for each participant and arm. A linear regression was performed for each plot. These plots include all subjects who received a device and attended interim or final assessment (i.e., even those who used the device less than the minimum compliance of 36 hours by the end of the 12-week treatment period). Data points are jittered for visibility. $r$ is listed as absolute value. 
A
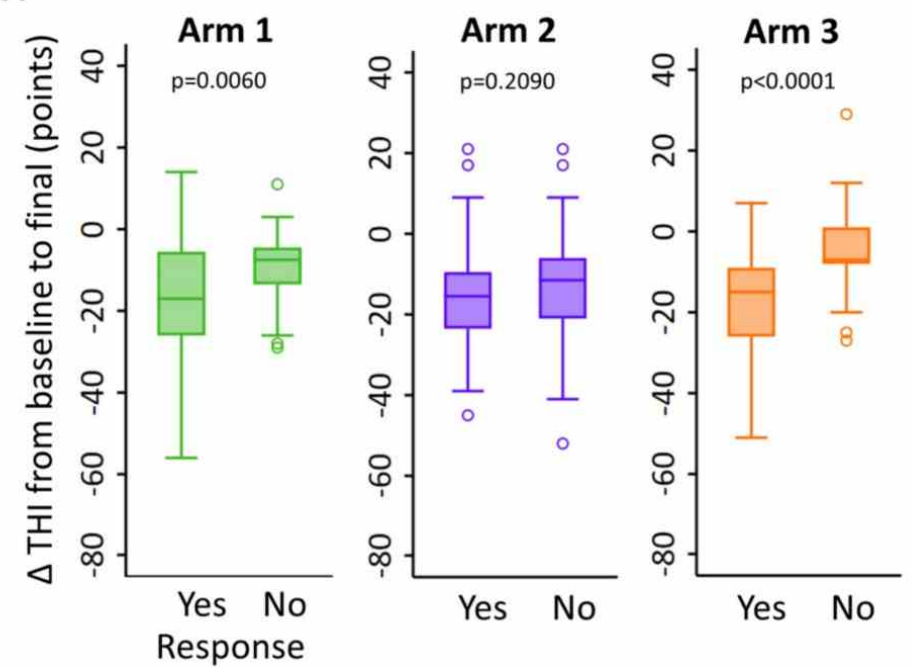

B
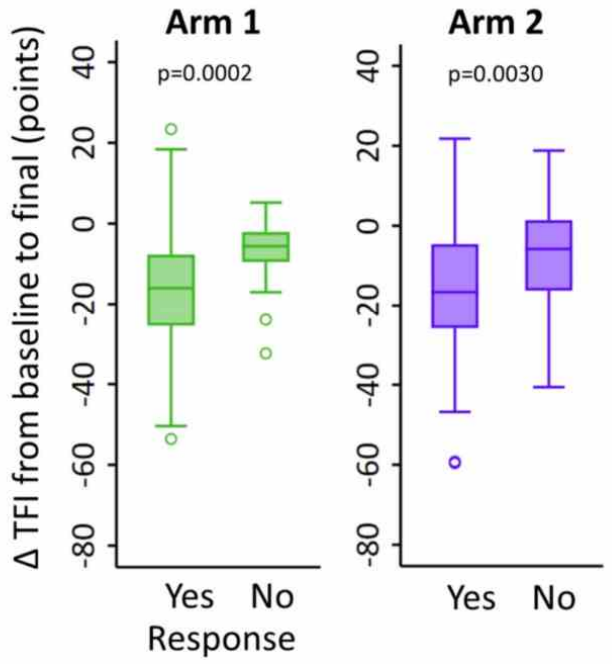

Arm 3

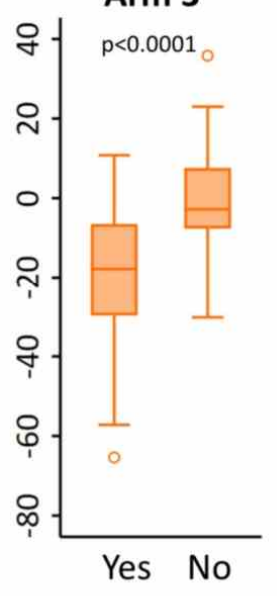

Fig. S12. Changes in tinnitus symptoms based on responses to first satisfaction question. Participant were asked at the end of treatment (fig. S1), "overall, would you say you have benefitted from using this device?" Out of 272 responses, $66.5 \%$ indicated "Yes". The change in (A) THI or (B) TFI score from baseline to final assessment for participants who responded "Yes" are plotted next to those who responded "No" for each arm with standard box plots. Statistical significance was determined using an unequal variance two-tailed t-test on ranked data appropriate for this dataset (77). 
A
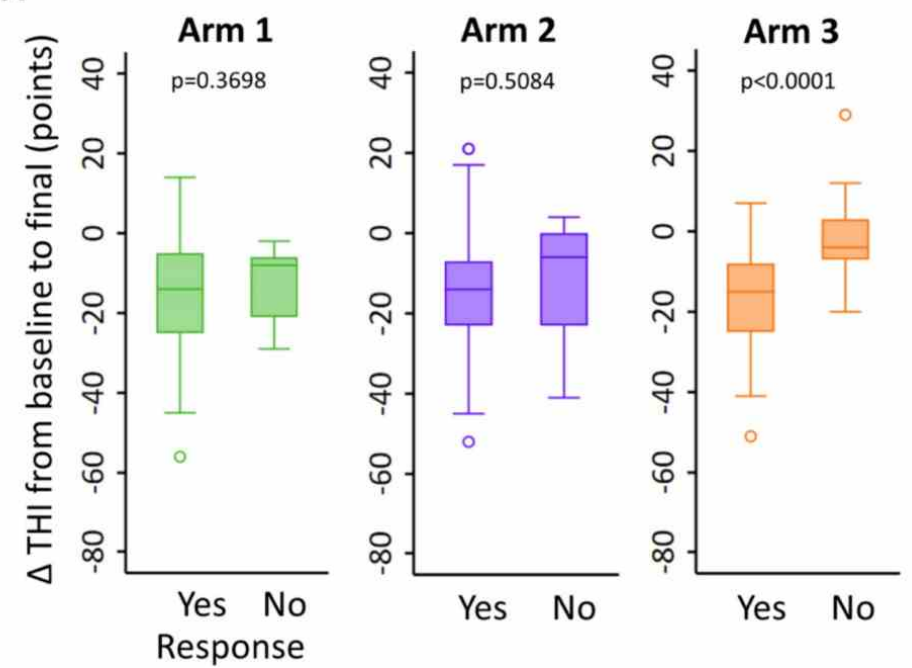

B
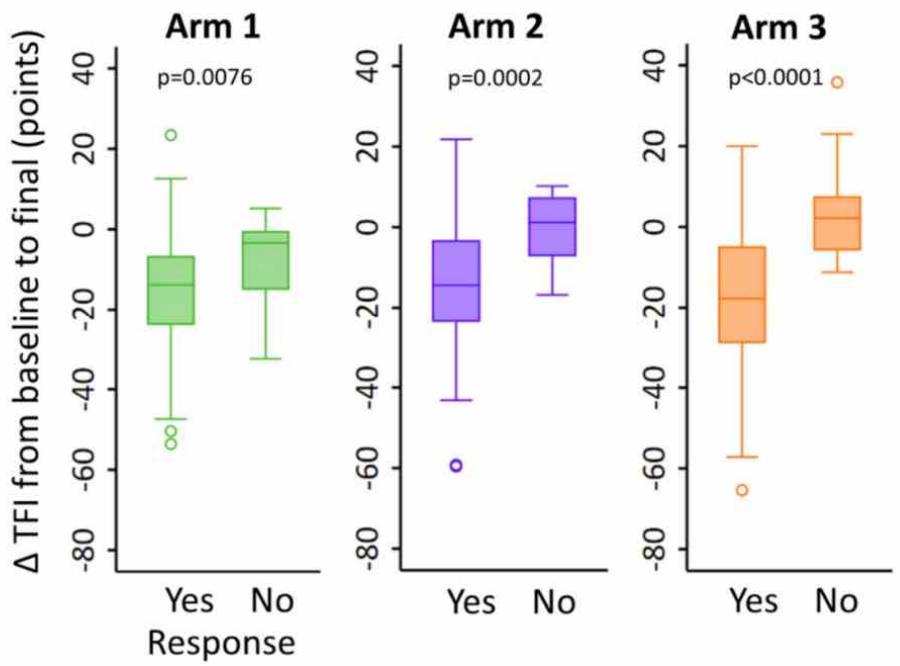

Fig. S13. Changes in tinnitus symptoms based on responses to second satisfaction question. Participants were asked at the end of treatment (fig. S1), "if you knew someone with tinnitus would you recommend they try this treatment?" Out of 270 responses, $77.8 \%$ indicated "Yes". The change in (A) THI or (B) TFI score from baseline to final assessment for participants who responded "Yes" are plotted next to those who responded "No" for each arm with standard box plots. Statistical significance was determined using an unequal variance two-tailed t-test on ranked data appropriate for this dataset (77). 

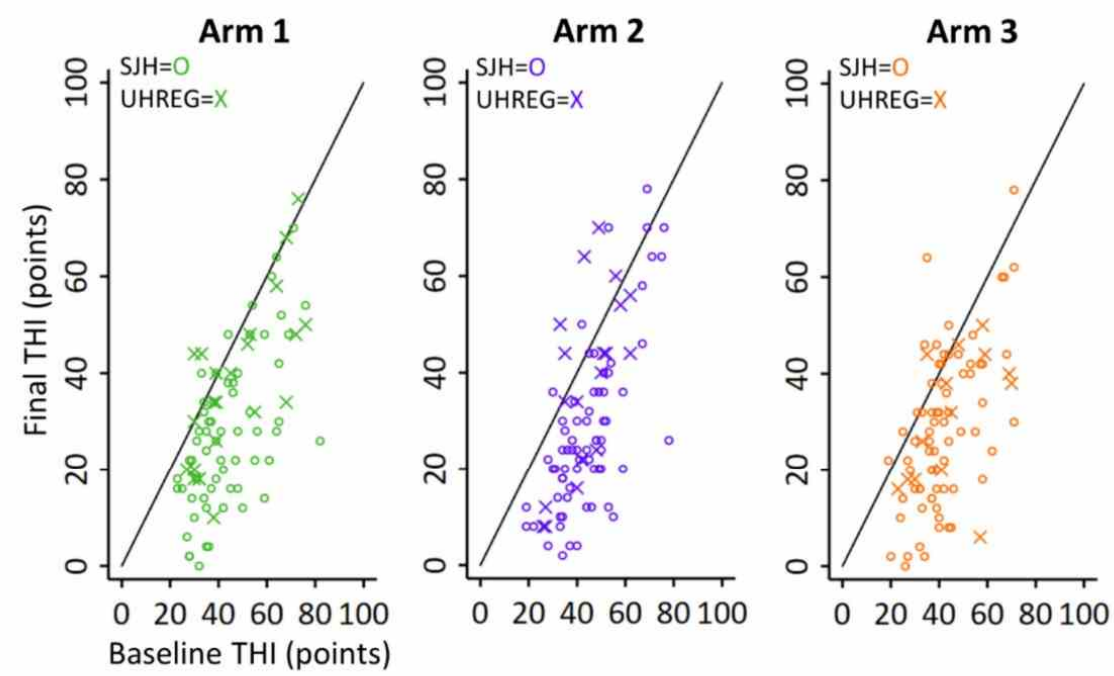

Fig. S14. Scatter plots for THI scores for each treatment-compliant individual at baseline versus end of treatment (12-week "final" visit) for each arm. The points are labeled based on clinical site (SJH: St. James's Hospital; UHREG: University Hospital Regensburg). Data points are jittered for visibility. 

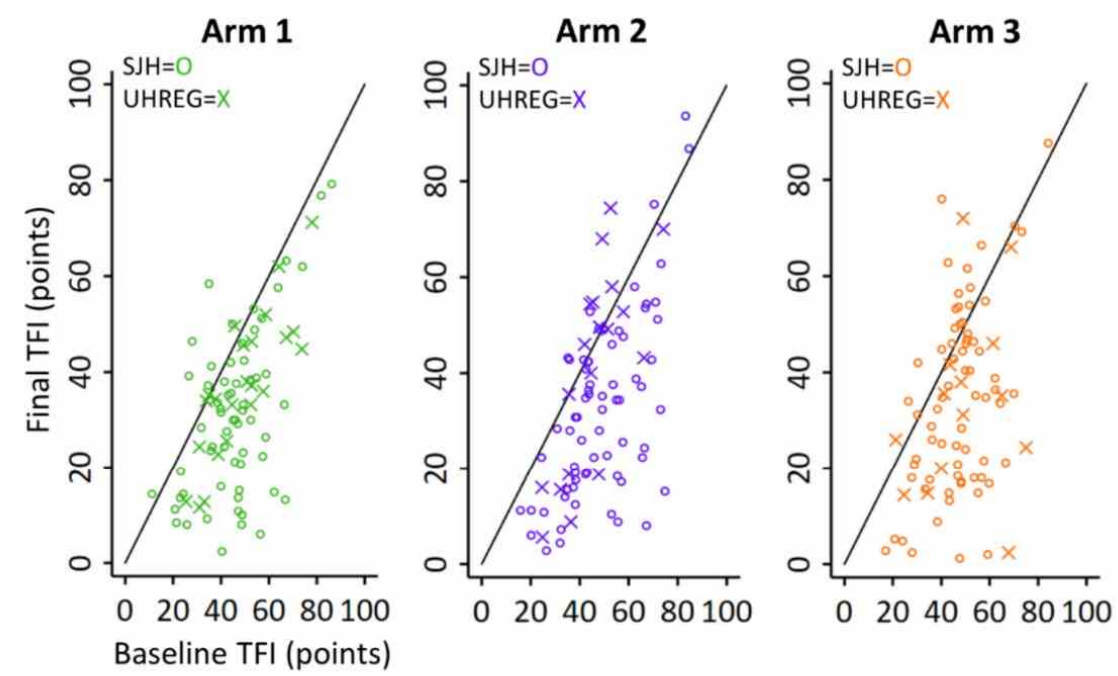

Fig. S15. Scatter plots for TFI scores for each treatment-compliant individual at baseline versus end of treatment (12-week "final" visit) for each arm. The points are labeled based on clinical site (SJH: St. James's Hospital; UHREG: University Hospital Regensburg). Data points are jittered for visibility. 
Table S1. Schematics and description of stimulation setting used in each arm.

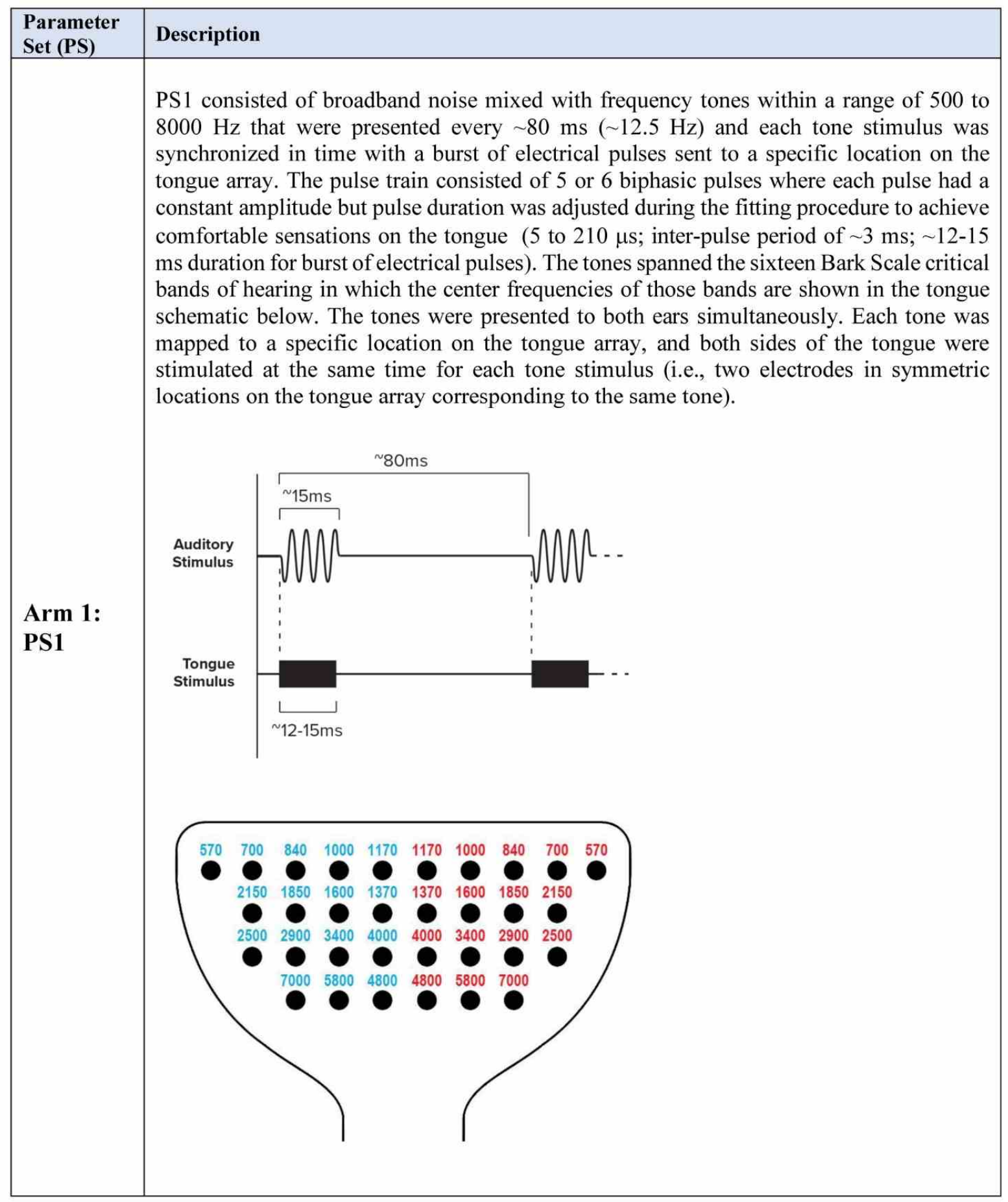




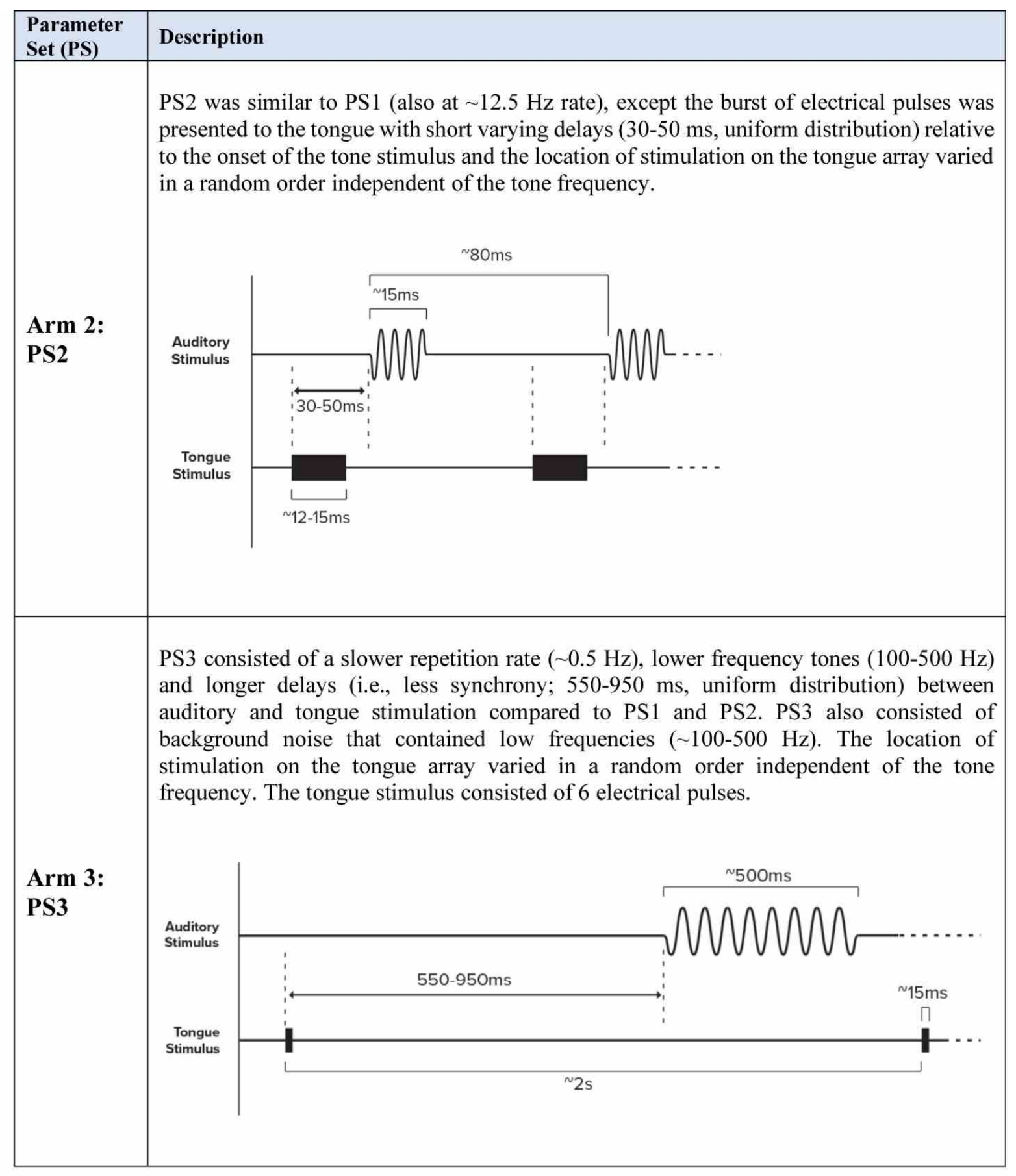


Table S2. Adverse events attributed to patient conditions from Table 2.

\begin{tabular}{|c|c|}
\hline Possibly device related: Negligible & Possibly device related: Moderate \\
\hline $\begin{array}{l}\text { - Itchiness on head from headphones but } \\
\text { participant reported they have sensitive skin } \\
\text { - Temporary stress with increased tinnitus but } \\
\text { participant indicated father passed away during } \\
\text { that time }\end{array}$ & $\begin{array}{l}\text { - Hearing loss in right ear ( } 30 \text { to } 40 \mathrm{~dB} \\
\text { increase at low frequencies of } 250,500 \text {, } \\
1000 \mathrm{~Hz} \text { ) with fullness/pressure in ears, but } \\
\text { previous experience with sudden hearing } \\
\text { loss possibly attributed to Meniere's disease }\end{array}$ \\
\hline Probably not device related: Negligible & Probably not device related: Moderate \\
\hline $\begin{array}{l}\text { - Cold sores but participant had history of cold } \\
\text { sores } \\
\text { - Pain in ear but also had concurrent ear infection } \\
\text { - Five cases of redness of the pharynx/throat but } \\
\text { attributed to colds or sore throat as likely cause } \\
\text { and these cases were not brought up during } \\
\text { treatment } \\
\text { - Two cases of small ulcers in the mouth but } \\
\text { participants reported biting themselves; these } \\
\text { ulcers were not bothersome } \\
\text { - Red eyes } \\
\text { - Sinus issue } \\
\text { - Developed tinnitus in right ear but also had } \\
\text { concurrent sinus issue } \\
\text { - Bumps at back of patients tongue but participant } \\
\text { was not aware of them } \\
\text { - Slight dizziness/disorientation but participant } \\
\text { was not sure } \\
\text { - Forgetfulness but unclear if related to device } \\
\text { - Sinusitis but participant has recurring condition } \\
\text { - Nausea but concurrently had Norovirus } \\
\text { - Common cold } \\
\text { - Difficulty in sleeping but participant reported } \\
\text { previous issues with sleeping } \\
\text { - Participant did not feel as fit during treatment } \\
\text { but also started a new medication for thyroid } \\
\text { gland issue } \\
\text { - Heart palpitations for a few seconds } \\
\text { body temporarily }\end{array}$ & $\begin{array}{l}\text { - Uncomfortable ulcer but participant } \\
\text { indicated that they bit down on mouth that } \\
\text { caused it } \\
\text { Breathing issues but participant has a } \\
\text { history of breathing issues and chronic } \\
\text { obstructive lung disease, and also } \\
\text { concurrently had a pulmonary infection } \\
\text { during the treatment period }\end{array}$ \\
\hline
\end{tabular}


Table S3. Patient referrals for adverse events. Of the adverse events listed in Table 2, there were three cases in which the participants were referred to an otolaryngologist or audiologist as described in the table below.

\begin{tabular}{|l|l|}
\hline Adverse event & Referral description \\
\hline $\begin{array}{l}\text { Increase in } \\
\text { tinnitus listed in } \\
\text { Table 2 }\end{array}$ & $\begin{array}{l}\text { Tinnitus symptoms increased in right ear since starting bimodal neuromodulation } \\
\text { treatment and was not reduced by the end of the treatment, which further } \\
\text { contributed to disruptions in sleeping. The individual reported an ear infection in } \\
\text { the right ear in the previous year before treatment that led to being admitted into } \\
\text { the hospital. This individual was referred to an otolaryngologist for further } \\
\text { assessment with no major clinical concerns reported. At the 6-month follow-up } \\
\text { assessment, the adverse event had resolved. }\end{array}$ \\
\hline $\begin{array}{l}\text { Increase in } \\
\text { tinnitus listed in } \\
\text { Table 2 }\end{array}$ & $\begin{array}{l}\text { Individual returned device after ending treatment and indicated tinnitus had } \\
\text { worsened since starting treatment. The individual also reported possible } \\
\text { fluctuations in hearing, fluctuations in tinnitus and dizziness. After a final review } \\
\text { assessment, the individual was referred to an audiologist to continue monitoring } \\
\text { symptoms beyond the study. }\end{array}$ \\
\hline $\begin{array}{l}\text { Recurring sudden } \\
\text { hearing loss listed } \\
\text { in table S2 } \\
\text { associated with } \\
\text { patient conditions }\end{array}$ & $\begin{array}{l}\text { Hearing loss in right ear (30 to 40 dB increase at low frequencies of 250, 500, } \\
\text { 1000 Hz) with fullness/pressure in ears, but previous experience with sudden } \\
\text { hearing loss possibly attributed to Meniere's disease. Individual also reported } \\
\text { tinnitus increased with changes in its pitch. The individual was referred to an } \\
\text { otolaryngologist who assessed and confirmed Meniere's disease. }\end{array}$ \\
\hline
\end{tabular}


Table S4. Stratification category of hyperacusis in Table 1 calculated in units of dB HL. In Table 1, the hyperacusis category (loudness discomfort level, LDL, at $500 \mathrm{~Hz}$ ) is calculated in units of dB SL (LDL $<60 \mathrm{~dB} \mathrm{SL}$ ). The table below calculates LDL in units of dB HL. Based on the audiogram data plotted in fig. S3, thresholds for $500 \mathrm{~Hz}$ can range from about 0 to $20 \mathrm{~dB}$ HL. Therefore, the distribution of participants across arms is provided in the table below for three different criteria in units of $\mathrm{dB}$ HL that aligns with the original LDL criterion that was in units of $\mathrm{dB}$ SL: LDL $<60 \mathrm{~dB}$ HL, LDL $<70 \mathrm{~dB}$ HL and LDL $<80 \mathrm{~dB}$ HL. There is no significant difference between arms for any of these criteria, based on the Fisher's exact test $(P>0.05)$.

\begin{tabular}{|c|c|c|c|c|c|c|}
\hline Characteristics & Units & Full cohort & Arm 1 & Arm 2 & Arm 3 & P-value \\
\hline $\begin{array}{c}\text { LDL }<60 \mathrm{~dB} \mathrm{HL} \\
\text { at } 500 \mathrm{~Hz}\end{array}$ & $\begin{array}{c}\text { \# participants } \\
\text { (\% of enrolled) }\end{array}$ & $7(2.1 \%)$ & $2(1.8 \%)$ & $3(2.8 \%)$ & $2(1.8 \%)$ & 1.000 \\
\hline $\begin{array}{c}\mathrm{LDL}<70 \mathrm{~dB} \mathrm{HL} \\
\text { at } 500 \mathrm{~Hz}\end{array}$ & $\begin{array}{c}\text { \# participants } \\
\text { (\% of enrolled) }\end{array}$ & $36(11.0 \%)$ & $11(10.0 \%)$ & $12(11.2 \%)$ & $13(11.9 \%)$ & 1.000 \\
\hline $\begin{array}{c}\text { LDL }<80 \mathrm{~dB} \mathrm{HL} \\
\text { at } 500 \mathrm{~Hz}\end{array}$ & $\begin{array}{c}\text { \# participants } \\
\text { (\% of enrolled) }\end{array}$ & $110(33.7 \%)$ & $37(33.6 \%)$ & $39(36.4 \%)$ & $34(31.2 \%)$ & 0.928 \\
\hline
\end{tabular}

